Biomath Communications

http://www.biomathforum.org/biomath/index.php/conference Biomath Forum

\title{
An Extension of Two Species Lotka-Volterra Competition Model
}

\author{
Idy $\mathrm{Ba}^{1,2}$, Papa Ibrahima Ndiaye ${ }^{1,2}, \mathrm{Mahe} \mathrm{Ndao}^{2}$, Aboubakary Diakhaby $^{2}$ \\ ${ }^{1}$ Université Alioune Diop, Bambey, Sénégal \\ idy.ba@uadb.edu.sn \\ papaibra.ndiaye@uadb.edu.sn \\ ${ }^{2}$ Université Gaston Berger, Saint-Louis, Sénégal \\ mahe16g2g@yahoo.fr \\ diakhaby@ugb.edu.sn
}

\begin{abstract}
Limiting resource is a angular stone of the interactions between species in ecosystems such as competition, prey-predators and food chain systems. In this paper, we propose a planar system as an extension of Lotka-Volterra competition model. This describes two competitive species for a single resource which are affected by intra and inter-specific interference. We give its complete analysis for the existence and local stability of all equlibria and some conditions of global stability. The model exhibits a rich set of behaviors with a multiplicity of coexistence equilibria, bi-stability, tri-stability and occurrence of global stability of the exclusion of one species and the coexistence equilibrium. The asymptotic behavior and the number of coexistence equilibria are shown by a saddle-node bifurcation of the level of resource under conditions on competitive effects relatively to associated growth rate per unit of resource. Moreover, we determine the competition outcome in the situations of Balanced and Unbalanced intra-inter species competition effects. Finally, we illustrate results by numerical simulations.
\end{abstract}

Keywords: consumer-resource model; invariant manifolds; competition outcome; asymptotic behavior

MSC2010: 92D40; 34K19; 35B40

Copyright: (C) 2021 Idy Ba, Papa Ibrahima Ndiaye, Mahe Ndao, Aboubakary Diakhaby. This article is distributed under the terms of the Creative Commons Attribution License 4.0, which permits unrestricted use, distribution, and reproduction in any medium, provided the original author and source are credited. Received: November 13, 2021, Accepted: November 25, 2021, Published: December 17, 2021, Updated: June 2, 2022

Citation: Idy Ba, Papa Ibrahima Ndiaye, Mahe Ndao, Aboubakary Diakhaby, An Extension of Two Species Lotka-Volterra Competition Model, Biomath Communications 8 (2021), 2112171, https://doi.org/10.11145/bmc.2021.12.171 


\section{Introduction}

In nature, organisms of a species members live in a area with other populations species and resources they need for their growth. When a large number of organisms affect other populations species by their presence or absence, in some cases of mixed populations species, two or more species interact in a food chain and prey-predator systems or compete for common resources such as food, light, space, etc. In the situation of two species compete with each other for the same resource, Hardin [1] argued that the Competitive Exclusion Principle (CEP) formulated by Gause in [2] holds, this means that the "better" competitor will eventually exclude the others. This principle has been formally demonstrated in [3] with classical LotkaVolterra competition (LV) model analysis and set to theory [1].

Numerous evidences based on laboratory experiences and natural observations are supporting the CEP. Among them, the complexity of competitive situations make unclear the predictive strength of the displayed species as Thomas Park and his collaborator experiments showed in a closed universe under various conditions [5]. Beyond this phenomena, the observed diversity of certain communities such as coexistence of phytoplankton species competing for very limiting resource is in apparent contradiction with Gause's law. This paradox was originally described in aquatic biology by Hutchinson in [6], who suggested that it could be resolved by factors such as vertical gradient of light or turbulence, symbiosis or commensalism, differential predation or sufficiently frequent variations of the environment.

The generalization of (LV) model to more than two species have led to deep ecological insights [7] and allowed to identify interesting ecological phases and phase transitions [8,9] without explain the phytoplankton paradox foundation. The implicit description of resources in play for two or more competing species in such model apparently hides the resources dynamics effects in competition outcome. Therefore, recent works have proposed models taken account chaotic fluid motion or water levels variations, spatio-temporal heterogeneity interaction with species to resolve the paradox [10]. These external factors are well known to impact the densities of resources or heterogeneity and availability.

The aim of this paper is to study the impact of resource level on competition outputs in the overview of the CEP. MacArthur [11, 12, 13, 14, 15] introduced first the class "consumer-resource models" which incorporate 
explicitly resource consummation in LV species equations.

MacArthur in [16] shows that a new species can compete on community if only its action of competition minimizes a quadratic function. Also, a large number of competing species can coexist if the productive environment and the environmental variations can compromise the CEP. Soon after, May and MacArthur in [17] tackle niches ecology overlap problem by combining their quadratic functions.

Nevertheless, P. Chesson in [18] considered both exploitative competition and interference competition in the model to generalize the MacArthur [16] results and revealed that the arguments of Roughgarden in [19] and [20] contrasted May and MacArthur [17] conclusions on community structure. Specifically, he precises and reinforces MacArthur's [16] developments in the understanding and the empirical measurements of LV coefficients that distinguish timescales of resources and consumers in interaction.

Qualitatively, the MacArthur formulae is related to the Lyapunov function which guarantied the global stability of the coexistence equilibrium of the model resource-consumer without necessarily having a biological significance. In the literature, there exists a wide variety of competition by interference which do not lead to a minimized quadratic function form and the need to develop new models $[19,20,21]$. But, in a largest view of competition modeling subject, some mathematical models including densitydependent accessibility description are developed in bioreactor, food chain and prey-predators systems [22, 23, 24, 25, 26, 27, 28, 29, 30, 31].

In this paper, we propose and study a two species competition model including explicit resource consumption and density-dependent accessibility in the perspective to keep the effects of resource level variations and usual parameters relations with biological meaning on the competition outcome. To shake to simplicity without loss generality, the resource is assumed to be available in permanent regime and its accessibility rate is density-dependent of competitive species. A short description of biological parameters, assumptions, model equations and preliminary results such as positive and bounded solutions are presented in section 2 . In section 3 , it is given preliminaries results on two species Lotka-Volterra competition model as an occurrence of the model according to balanced and unbalanced inhibitory effects of two species intra and inter-specific inferences. The existence and stability analyses of coexistence and exclusion of one species equilibria of the model are presented. Thereafter, an application of the general theoret- 
ical results obtained in section 3 in the circumstances of "Balanced" and "Unbalanced" inhibitory effects conditions is devoted to section 4. Section 5 presents some numerical illustrations of analytical results.

\section{The Mathematical Model}

We consider two species living together in a area with carrying capacity $K$ and consuming a single limiting resource. From James Mallet [32] findings the carrying capacity is considered here to depend on habitat conditions. We assume that the growth rate of each population is inhibited by members both of its own species and of the other species as well as the accessibility of resource is affected by the presence of the other species. Also, members of each population are assumed to develop independently from each other. Let $t$ be a fixed time, we consider the density $x_{i}(t)$ and $x_{j}(t)$ of individuals of species $i$ and species $j$ respectively and denote by $Z, r_{i}^{\prime}$ and $s_{j i}$ the amount of available resource, the effective growth rate per unit of resource consumption of species $i$ and the rate to which the presence of species $j$ members affects the growth of species $i$ respectively. In addition, the food accessibility factor of species $i$ constrained by the presence of members of species $j$ is supposed to be $1 /\left(1+p_{j} x_{j}(t)\right)$ where the proportion $p_{j}$ is assumed constant. According to their assumptions, we describe the two species competition consuming a single limiting resource by the following system of equations.

$$
\left\{\begin{array}{l}
\frac{d x_{i}}{d t}=\left(\frac{r_{i}^{\prime} Z x_{i}}{1+p_{j} x_{j}}\right)-s_{i i} x_{i}^{2}-s_{i j} x_{i} x_{j}, \\
\frac{d x_{j}}{d t}=\left(\frac{r_{j}^{\prime} Z x_{j}}{1+p_{i} x_{i}}\right)-s_{j j} x_{j}^{2}-s_{j i} x_{i} x_{j},
\end{array}\right.
$$

For simplicity and without loss generality, the following model is considered throughout this paper

$$
\left\{\begin{array}{l}
\frac{d x_{i}}{d t}=\left(\frac{r_{i}^{\prime} Z x_{i}}{1+x_{j}}\right)-s_{i i} x_{i}^{2}-s_{i j} x_{i} x_{j}, \\
\frac{d x_{j}}{d t}=\left(\frac{r_{j}^{\prime} Z x_{j}}{1+x_{i}}\right)-s_{j j} x_{j}^{2}-s_{j i} x_{i} x_{j},
\end{array}\right.
$$


with initial conditions $x_{i}(0)=x_{0 i} \geq 0$ and $x_{j}(0)=x_{0 j} \geq 0$.

This model parameters and variables definitions are listed in table 1. In addition, we assume for parameters that $r_{i}^{\prime}>0, r_{j}^{\prime}>0, s_{i i}>0, s_{j j}>0$, $s_{j i}>0, s_{i j}>0, K>0,0 \leq Z<\frac{s}{r} K$ and $s=\min \left\{s_{i i}, s_{j j}, s_{j i}, s_{i j}\right\}$, $r=\max \left\{r_{i}^{\prime}, r_{j}^{\prime}\right\}$ and consider the domain of biological interest.

$$
\Omega=\left\{(x, y) \in \mathbb{R}_{+}^{2}, x+y \leq K\right\} .
$$

The well-posedness of the model (2) is then given by the next theorem.

Theorem 2.1. Assuming that the initial conditions lie in $\Omega$, the system of equations for two competitive species consuming a single limiting resource model (2) has a unique solution that exists and remains in $\Omega$ for all time $t \geq 0$.

Proof. The right-hand side of Equation (2) is continuous with continuous partial derivatives in $\Omega$, so the system of equation (2) has a unique solution. We now show that $\Omega$ is forward-invariant. If $x_{i}=0$ then $\frac{d x_{i}}{d t}=0$ and the system (2) is reduce to one species $j$ equation:

$$
\frac{d x_{j}}{d t}=r_{j}^{\prime} Z x_{j}\left(1-\frac{s_{j j}}{r_{j} Z} x_{j}\right)
$$

It's known that $\left(x_{j}\right.$-axis $)$ is invariant such that $\frac{d x_{j}}{d t}>0$ if only if $x_{j}>K_{j}$ when $Z>0$. If $x_{i}+x_{j}=K$ then

$$
\begin{aligned}
\frac{d x_{i}}{d t}+\frac{d x_{j}}{d t} & =\frac{r_{i}^{\prime} x_{i} Z}{1+x_{j}}+\frac{r_{j}^{\prime} x_{j} Z}{1+x_{i}} \\
& -s_{i i} x_{i}^{2}-s_{j j} x_{j}^{2}-s_{j i} x_{i} x_{j}-s_{i j} x_{i} x_{j} .
\end{aligned}
$$

This implies

$$
\frac{d x_{i}}{d t}+\frac{d x_{j}}{d t} \leq \frac{r_{i}^{\prime} x_{i} Z}{1+x_{j}}+\frac{r_{j}^{\prime} x_{j} Z}{1+x_{i}}-s x_{i}^{2}-s x_{j}^{2}-2 s x_{i} x_{j}
$$

from the assumption $s=\min \left\{s_{i i}, s_{j j}, s_{j i}, s_{i j}\right\}$. So, it results that

$$
\frac{d x_{i}}{d t}+\frac{d x_{j}}{d t} \leq K(r Z-s K)<0
$$

from hypothesis $0<Z<\frac{s}{r} K$ and $r=\max \left\{r_{i}^{\prime}, r_{j}^{\prime}\right\}$. Therefore, none of the orbits can leave $\Omega$ and a unique solution exists for all time $t \geq 0$. 
Table 1: Biological meanings of parameters used in the system (1) and their units.

\begin{tabular}{lll}
\hline Parameters & Descriptions & units \\
\hline$t$ & date & $(t)$ (time) \\
$Z$ & density of resource & $\mathcal{R}$ (resource) \\
$x_{i}$ & density of the species $i$ over time & $\mathcal{B}$ (biomass) \\
$r_{i}^{\prime}$ & Consumption rate per unit of resource & $(t \mathcal{R})^{-1}$ \\
$s_{i i}$ & nuisance of the species $i$ on itself per unit of & $(t \mathcal{B})^{-1}$ \\
& time and biomass & \\
$s_{i j}$ & nuisance of the species $j$ on species $i$ per unit & $(t \mathcal{B})^{-1}$ \\
& of time and biomass & \\
$p_{i}$ & fraction of species $j$ affecting resource acces- & percent \\
& sibility of an individual of species $i$ & \\
\hline
\end{tabular}

The first part of this proof see equation (3) provides the following useful remark.

Remark 2.1. As it was found for the Lotka-Volterra system (4), it states that in the absence of one species the system (2) is reduced to the logistic equation describing the living species when the limiting resource is available and accessible for its species. For instance, if $x_{i}=0$ the system (2) is reduced (3) with $r_{j}=r_{j}^{\prime} Z$ as growth rate and $K_{j j}=r_{j}^{\prime} Z / s_{j j}$ as its limit growth when $Z>0$.

\section{Mathematical analysis}

In this section, we determine the model (2) equilibria and study their local and global asymptotic stability.

\subsection{Lotka-Volterra-MacArthur model outcomes: Competitive effects relations}

In the literature, two competitive species models outcomes are considered in the sense of asymptotic behaviors of the system under study. Particularly, the developments in this subsection recall and focus on existing 
equilibria asymptotic stability results according to usual relations between parameters of the model with biological sense.

The following types of equilibria have been intensively studied:

- $E_{0}=(0,0)$ called the extinction equilibrium where both species populations are extinct: $x_{i}=x_{j}=0$.

- $E_{i}=\left(K_{i i}, 0\right)$ called the exclusion of species $j$ equilibrium where species $j$ population is excluded: $x_{i}>0$ and $x_{j}=0$.

- $E_{j}=\left(0, K_{j j}\right)$ called the exclusion of species $i$ equilibrium where species $i$ population is excluded: $x_{i}=0$ and $x_{j}>0$.

- $E^{*}=\left(x_{i}^{*}, x_{j}^{*}\right)$ called a coexistence equilibrium, where both species populations survive: $x_{i}^{*}>0$ and $x_{j}^{*}>0$.

Note that in the $p_{i} \rightarrow 0, p_{j} \rightarrow 0$ limits,

$r_{i}=r_{i}^{\prime} Z>0$ and $r_{j}=r_{j}^{\prime} Z>0$ the model (1) is reduced to the classic Lotka-volterra competition model in [3].

$$
\left\{\begin{array}{l}
\dot{x}_{i}=x_{i}\left(r_{i}-s_{i i} x_{i}-s_{i j} x_{j}\right) \\
\dot{x}_{j}=x_{j}\left(r_{j}-s_{j i} x_{i}-s_{j j} x_{j}\right)
\end{array}\right.
$$

In this case, the trivial equilibria $E_{0}, E_{i}$, and $E_{j}$ always exist. But, as it's well known, the coexistence equilibrium existence and equilibria stability of the system (4) depend on relations between expressions $t_{k l}=s_{k l} / r_{k}$, $(k, l \in\{i, j\})$ defined as the competitive effect of species $l$ on species $k$ relative to the growth rate of species $k$. Under these notations, the following four distinguishable cases were often considered:

(a) $t_{i i}>t_{j i}$ and $t_{i j}<t_{j j}$

(b) $t_{i i}<t_{j i}$ and $t_{i j}<t_{j j}$

(c) $t_{i i}>t_{j i}$ and $t_{i j}>t_{j j}$

(d) $t_{i i}<t_{j i}$ and $t_{i j}>t_{j j}$

Some of these conditions, for instance (b), are known to hide an helpful supplementary condition (underlining by Renshaw in [33]) that is the product 
of the within species inhibitory growth rates equals the product of the between-species inhibitory growth rates, i.e.,

$$
t_{i i} t_{j j}=t_{i j} t_{j i}
$$

Under only this additional condition (5), the system (4) can be rewritten in the following form

$$
\left\{\begin{array}{l}
\dot{x}_{i}=x_{i}\left[r_{i}-s_{i i}\left(x_{i}+q x_{j}\right)\right] \\
\dot{x}_{j}=x_{j}\left[r_{j}-s_{j i}\left(x_{i}+q x_{j}\right)\right]
\end{array}\right.
$$

where the constant $q\left(q=s_{i j} / s_{i i}=s_{j j} / s_{j i}\right)$ can describe the population of species $j$ make smaller demands on available resources when $q<1$ and otherwise $q>1$. From this system (6), three distinguishable biological behaviors are shown in [33] for any initial condition $\left(x_{0 i}, x_{0 j}\right)$ :

- If $K_{i i}>K_{j j}$, then $\lim _{t \rightarrow+\infty} x_{j}(t)=0$, i.e, the species $i$ wins the competition.

- If $K_{i i}<K_{j j}$, then $\lim _{t \rightarrow+\infty} x_{i}(t)=0$, i.e, the species $j$ wins the competition.

- If $K_{i i}=K_{j j}$, then neither $\lim _{t \rightarrow+\infty} x_{j}(t)=0$ nor $\lim _{t \rightarrow+\infty} x_{i}(t)=0$, i.e, the two species coexist.

Moreover, it stated in [3] that:

- if case (a) holds then the two species coexist;

- if case (b) holds then species $i$ wins the competition;

- if case (c) holds then species $j$ wins the competition;

- if case (d) holds the species $i$ or $j$ wins the competition with basin of attraction separated by the stable manifold of the coexistence equilibrium.

Throughout this work, the condition (5) is called "balanced intra-interspecific competition effects" condition as well as when it is not considered the 
term "balanced" is replaced by the word "unbalanced". So, there are two distinct "unbalanced intra-interspecific competition effects" conditions:

$$
t_{i i} t_{j j}>t_{i j} t_{j i}
$$

and

$$
t_{i i} t_{j j}<t_{i j} t_{j i}
$$

According to expressions of competitive effects relatively to growth rate, notice that when $Z>0$, the difference between $r_{k}$ and $r_{k}^{\prime}$ is displayed in the conditions (5), (7) and (8) as well as in the cases (a), (b), (c) and (d). In addition, two species Lotka-Volterra competition model (4) and its MacArthur Model [14] equivalent form that suppose unrestrained resource accessibility for each species:

$$
\left\{\begin{array}{l}
\dot{x}_{i}=r_{i}^{\prime} Z x_{i}\left(1-t_{i i} x_{i}-t_{i j} x_{j}\right) \\
\dot{x}_{j}=r_{j}^{\prime} Z x_{j}\left(1-t_{j i} x_{i}-t_{j j} x_{j}\right),
\end{array}\right.
$$

have the same conclusions (see e.g $[3,4]$ ).

In the further pursuit of theoretical and numerical studies of the model (2), we consider the definitions, the relations and the notations introduced in this current subsection. Moreover, we will use, for convenience, the abbreviations (LAS) for Locally Asymptotically Stable equilibria (U) for Unstable equilibria and (GAS) for Globally Asymptotically Stable equilibria in all the following.

\subsection{General properties of the model}

In this subsection, we consider unavailable and available of the limiting resource conditions to study the general properties of the model (2).

Theorem 3.1. If $Z=0$ then the system (2) admits an unique equilibrium point $E_{0}=(0,0)$ which is globally asymptotically stable. Else, for any $Z>0$ the extinction equilibrium $E_{0}=(0,0)$ is a repeller point of the system (2).

Proof. First, if $Z=0$ then $E_{0}=(0,0)$ is the only equilibrium point of the system (2). To show its global asymptotic stability, let $V: \mathbb{R}_{+}^{2} \longrightarrow \mathbb{R}_{+}$the lyapunov function defined by $V\left(x_{i}, x_{j}\right)=x_{i}+x_{j} . V$ is obviously $\mathcal{C}^{1}\left(\mathbb{R}_{+}^{2}\right)$ and satisfies the following conditions: 


$$
\begin{aligned}
& V(0,0)=0, \forall\left(x_{i}, x_{j}\right) \neq(0,0), \\
& V\left(x_{i}, x_{j}\right)>0, \\
& \dot{V}\left(x_{i}, x_{j}\right)=\dot{x}_{i}+\dot{x}_{j}<0 \text { and } \\
& V\left(x_{i}, x_{j}\right) \longrightarrow \infty \text { as }\left\|\left(x_{i}, x_{j}\right)\right\| \longrightarrow \infty .
\end{aligned}
$$

Thus, by Barbashin \& Krasovskii [34], the origin is globally asymptotically stable for the system (2).

Second, let's suppose $Z>0$, then the Jacobian Matrix of the system (2) at any point $\left(x_{i}, x_{j}\right) \in \Omega$ is

$$
J\left(x_{i}, x_{j}\right)=\left(\begin{array}{ll}
j_{1}\left(x_{i}, x_{j}\right) & j_{3}\left(x_{i}, x_{j}\right) \\
j_{4}\left(x_{i}, x_{j}\right) & j_{2}\left(x_{i}, x_{j}\right)
\end{array}\right)
$$

where

$$
\begin{aligned}
& j_{1}\left(x_{i}, x_{j}\right)=\frac{r_{i} Z}{1+x_{j}}-2 s_{i i} x_{i}-s_{i j} x_{j}, \\
& j_{3}\left(x_{i}, x_{j}\right)=-\frac{r_{i} Z x_{i}}{\left(1+x_{j}\right)^{2}}-s_{i j} x_{i}, \\
& j_{4}\left(x_{i}, x_{j}\right)=-\frac{r_{j} Z x_{j}}{\left(1+x_{i}\right)^{2}}-s_{j i} x_{j} \text { and } \\
& j_{2}\left(x_{i}, x_{j}\right)=\frac{r_{j} Z}{1+x_{i}}-2 s_{j j} x_{j}-s_{j i} x_{i} .
\end{aligned}
$$

Particularly, the Jacobian matrix (10) at $E_{0}$ has two positive eigenvalues $r_{i} Z>0$ and $r_{j} Z>0$. Therefore, $E_{0}$ is an unstable and repeller point for the system (2).

Remark 3.1. The results of the theorem 3.1 corresponds to two species extinction scenario in the absence of resource $(Z=0)$ while the presence $(Z>0)$ of accessible limiting resource leads to competitive exclusion principle or coexistence of two species outcome.

Now, since the asymptotic behavior of the model is summarized in theorem 3.1 when $Z=0$, it is then supposed throughout in the rest of this paper that two species live both at the beginning of the experiment and consume permanent limiting resource $(Z>0)$ thereafter. 
Lemma 3.1. Let $Z>0$ and $K_{s}=\max _{k, l \in\{i, j\}}\left\{\frac{r_{k} Z}{s_{k l}}\right\}$. Then the set:

$$
\Omega_{s}=\left\{\left(x_{i}, x_{j}\right) \in \mathbb{R}_{+}^{2} ; x_{i}+x_{j} \leq K_{s}\right\}
$$

is a global attractor for the system (2). Moreover, the extinction Equilibrium $E_{0}=(0,0)$, the exclusion of one species equilibria $E_{i}=\left(K_{i i}, 0\right)$ and $E_{j}=\left(0, K_{j j}\right)$ exist and lie all in $\Omega_{s}$

Proof. It's obvious that $\Omega_{s}$ is a compact set and invariant for the system (2) by the main ideas developed in the proof of the Theorem 2.1. Thus, it remains to show that $\Omega_{s}$ attracts any neighboring trajectory starting in $\Omega$. Let $P=\left(x_{i}, x_{j}\right) \in \Omega$ so that $x_{i}+x_{j}>K_{s}, i . e, 1-\frac{x_{i}+x_{j}}{K_{s}}<0$. Therefore, from the inequalities

$$
\frac{d x_{i}}{d t} \leq r_{i} Z x_{i}\left(1-\frac{x_{i}}{K_{i i}}-\frac{x_{j}}{K_{i j}}\right) \leq r_{i} Z x_{i}\left(1-\frac{x_{i}+x_{j}}{K_{s}}\right)<0
$$

and similarly for $x_{j}$, it results $\frac{d x_{i}}{d t}+\frac{d x_{j}}{d t}<0$. By setting $\dot{x}_{i}=0$ and $x_{j}=0$, we have $x_{i}=\frac{r_{i} Z}{s_{i i}}$, i.e, $E_{i}$ is an equilibrium point for (2). This completes the proof.

Lemma 3.2. There is no periodic orbit for the system (2) lying entirely in the interior of $\Omega$.

Proof. Consider for any point $P=\left(x_{i}, x_{j}\right)$ in the interior of the bounded set $\Omega$, the functions $B\left(x_{i}, x_{j}\right)=\frac{1}{x_{i} x_{j}}$ and

$$
f\left(x_{i}, x_{j}\right)=\left(\begin{array}{l}
r_{i} Z\left(\frac{1}{1+x_{j}}\right) x_{i}-s_{i i} x_{i}^{2}-s_{i j} x_{i} x_{j}, \\
r_{j} Z\left(\frac{1}{1+x_{i}}\right) x_{j}-s_{j j} x_{j}^{2}-s_{j i} x_{i} x_{j}
\end{array}\right) .
$$

Then, by calculation, one obtains the divergence of $B f$ :

$$
\operatorname{div}\left(B f\left(x_{i}, x_{j}\right)\right)=-\left(\frac{s_{i i}}{x_{j}}+\frac{s_{j j}}{x_{i}}\right)<0 .
$$

Hence, by Lemma 3.1 and Dulac criteria (see [35]), there can be no closed orbit entirely in the interior of $\Omega$. This ends the proof. 
The above Lemma 3.2 will be used in the proof of our global asymptotic stability result of exclusion or coexistence equilibrium.

\subsection{Coexistence equilibrium point}

It's obvious that a so called equilibrium of coexistence $E^{*}=\left(x_{i}^{*}, x_{j}^{*}\right)$ $\left(x_{i}^{*}>0\right.$ and $\left.x_{j}^{*}>0\right)$ of the system (2) satisfies the following system of equations

$$
\left\{\begin{array}{l}
\frac{r_{i} Z}{1+x_{j}^{*}}-s_{i i} x_{i}^{*}-s_{i j} x_{j}^{*}=0, \\
\frac{r_{j} Z^{*}}{1+x_{i}^{*}}-s_{j j} x_{j}^{*}-s_{j i} x_{i}^{*}=0 .
\end{array}\right.
$$

Then, after little algebra from (11), a useful necessary condition for being a coexistence equilibrium $E^{*}$ states in the following Lemma.

Lemma 3.3. Let $Z>0$. If $E^{*}=\left(x_{i}^{*}, x_{j}^{*}\right)$ is a coexistence equilibrium then these coordinates $\left(x_{i}^{*}, x_{j}^{*}\right)$ satisfy the following set of equations:

$$
\begin{aligned}
& a_{4}\left(x_{i}^{*}\right)^{4}+a_{3}\left(x_{i}^{*}\right)^{3}+a_{2}\left(x_{i}^{*}\right)^{2}+a_{1} x_{i}^{*}+a_{0}=0, \\
& a_{4}^{\prime}\left(x_{j}^{*}\right)^{4}+a_{3}^{\prime}\left(x_{j}^{*}\right)^{3}+a_{2}^{\prime}\left(x_{j}^{*}\right)^{2}+a_{1}^{\prime} x_{j}^{*}+a_{0}^{\prime}=0,
\end{aligned}
$$

where

- $a_{4}=s_{j i} \alpha_{c}, a_{4}^{\prime}=-s_{i j} \alpha_{c}$ with $\alpha_{c}=s_{i i} s_{j j}-s_{j i} s_{i j}$,

- $a_{3}=\left(2 s_{j i}-s_{j j}\right) \alpha_{c}, a_{3}^{\prime}=-\left(2 s_{i j}-s_{i i}\right) \alpha_{c}$

- $a_{2}=e_{i} Z+\left(-2 s_{j j}-r_{j}^{\prime} Z+s_{j i}\right) \alpha_{c}$, $a_{2}^{\prime}=e_{i}^{\prime} Z-\left(-2 s_{i i}-r_{i}^{\prime} Z+s_{i j}\right) \alpha_{c}$ where $e_{i}=s_{j j}^{2} r_{i}^{\prime}+s_{j i} s_{i j} r_{j}^{\prime}>0$ and $e_{i}^{\prime}=s_{i i}^{2} r_{j}^{\prime}+s_{i j} s_{j i} r_{i}^{\prime}>0$,

- $a_{1}=e_{i} Z-\left(s_{j j}+r_{j}^{\prime} Z\right) \alpha_{c}+d_{i} Z$, $a_{1}^{\prime}=e_{i}^{\prime} Z+\left(s_{i i}+r_{i}^{\prime} Z\right) \alpha_{c}+d_{i}^{\prime} Z$ where $d_{i}=s_{i j} r_{j}^{\prime 2} Z_{c_{i}}$ and $d_{i}^{\prime}=s_{j i} r_{i}^{\prime 2} Z_{c_{j}}$

- $a_{0}=s_{i j} r_{j}^{\prime 2}\left(Z_{c_{i}}-Z\right) Z, a_{0}^{\prime}=s_{j i} r_{i}^{\prime 2}\left(Z_{c_{j}}-Z\right) Z$

with $Z_{c_{i}}=\frac{s_{i i}}{r_{i}^{\prime}}\left(\frac{t_{i i}}{t_{j i}}-1\right)$ and $Z_{c_{j}}=\frac{s_{j j}}{r_{j}^{\prime}}\left(\frac{t_{j j}}{t_{i j}}-1\right)$. 
Proof. See Appendix A.

Remark 3.2. Since the polynomial coefficients $a_{4}$ and $a_{4}^{\prime}$ are opposite sign as well as $a_{3}$ and $a_{3}^{\prime}$ do, the study of the corresponding Polynomial's roots may be equivalent.

For analytical pursuit of coexistence equilibrium studies, the system (11) can be rewritten as

$$
\left\{\begin{array}{l}
0=x_{i}\left(\phi_{i}\left(x_{i}\right)-f_{i}\left(x_{j}\right)\right) \\
0=x_{j}\left(\phi_{j}\left(x_{j}\right)-f_{j}\left(x_{i}\right)\right)
\end{array}\right.
$$

where for $k, l \in\{i, j\}$

$$
\phi_{k}(\tau)=r_{k}\left(1-\frac{\tau}{K_{k k}}\right), \quad f_{k}(\tau)=r_{k}\left(1-\frac{1}{1+\tau}+\frac{\tau}{K_{k l}}\right)
$$

with $r_{k}=r_{k}^{\prime} Z, \forall k \neq l$.

In the system $(14), \phi_{k}:\left[0 ;+\infty[\rightarrow]-\infty ; r_{k}\right]$ is continuous, straight line, decreasing and invertible function satisfying $\phi_{k}(0)=r_{k}, \phi_{k}\left(K_{k k}\right)=0$, $\phi_{k}(\tau) \geq 0$ iff $\tau \leq K_{k k}$ and $\forall \alpha \leq r_{k}, \phi_{k}^{-1}(\alpha)=K_{k k}\left(1-\frac{\alpha}{r_{k}}\right)$.

Moreover, $f_{k}:[0,+\infty[\rightarrow[0,+\infty[$ is continuous, increasing, concave $\left(f_{k}^{\prime \prime}(\tau)<0, \forall \tau \geq 0\right)$ and invertible function. Therefore, the system (14) becomes

$$
\left\{\begin{array}{l}
x_{i}=g_{i}\left(x_{j}\right) \\
x_{i}=g_{j}\left(x_{j}\right)
\end{array}\right.
$$

where

$$
\begin{aligned}
& g_{i}=\phi_{i}^{-1} \circ f_{i}:\left[0, x_{j}^{\max }\right] \rightarrow\left[0, K_{i i}\right] \text { and } \\
& g_{j}=f_{j}^{-1} \circ \phi_{j}:\left[0, K_{j j}\right] \rightarrow\left[0, r_{j}\right]
\end{aligned}
$$

are both convex, strictly decreasing and continuous functions.

The properties of these functions (16) will be useful in the study of coexistence equilibrium point. For instance, it will be used in the proof of the following lemma which gives upper bounds of $E^{*}$ 's coordinates. 
Lemma 3.4. Let $Z>0$. A necessary condition for $E^{*}=\left(x_{i}^{*}, x_{j}^{*}\right)$ being a coexistence equilibrium of the system (2) is that

$$
\left.\left(x_{i}^{*}, x_{j}^{*}\right) \in\right] 0, \min \left(K_{i i}, x_{i}^{\max }\right)[\times] 0, \min \left(K_{j j}, x_{j}^{\max }\right)[
$$

where

$$
x_{i}^{\max }=\frac{-1+\sqrt{1+4 K_{j i}}}{2}
$$

and

$$
x_{j}^{\max }=\frac{-1+\sqrt{1+4 K_{i j}}}{2} .
$$

Proof. Let $Z>0$. Since $g_{i}$ is continuous, strictly decreasing and $g_{i}(0) g_{i}(K)$ $<0$, there exists an unique $\left.x_{j}^{\max } \in\right] 0, K$ [ solution of $g_{i}(\tau)=0$, equivalently, $f_{i}\left(x_{j}^{\max }\right)=r_{i}$. By solving this last equation, one can obtain:

$$
x_{j}^{\max }=\frac{-1+\sqrt{1+4 K_{i j}}}{2}
$$

and thereafter

$$
g_{i}:\left[0, x_{j}^{\max }\right] \rightarrow\left[0, K_{i i}\right]
$$

is invertible since

$$
\phi_{i}:\left[0, K_{i i}\right] \rightarrow\left[0, r_{i}\right]
$$

is positive and invertible. But, the coordinates of a coexistence equilibrium $E^{*}=\left(x_{i}^{*}, x_{j}^{*}\right)$ must be the solution of (15), i.e, $x_{i}^{*}=g_{i}\left(x_{j}^{*}\right)$ with $x_{i}^{*}>0$ and $x_{j}^{*}>0$. Therefore, since $g_{i}:\left[0 ; x_{j}^{\max }\right] \rightarrow\left[0 ; K_{i i}\right]$ is invertible, it results that $\left.x_{j}^{*}=g_{i}^{-1}\left(x_{i}^{*}\right) \in\right] 0, \min \left(K_{j j}, x_{j}^{\max }\right)[$.

In the same manner, we obtain $\left.x_{i}^{*} \in\right] 0, \min \left(K_{i i}, x_{i}^{\max }\right)[$. This completes this proof.

As it's well known, an intersection of nullclines curves in the interior of the domain $\Omega$ represents a coexistence equilibrium existence and one species exclusion equilibrium $E_{i}$ or $E_{j}$ is represented by the intersection point between a nullclines curves (11) and $\left(x_{i}-\right.$ axis $)$ or $\left(x_{j}-\right.$ axis $)$, the nullclines properties give typical situations as it is depicted in Figure 1 and Figure 2 . 

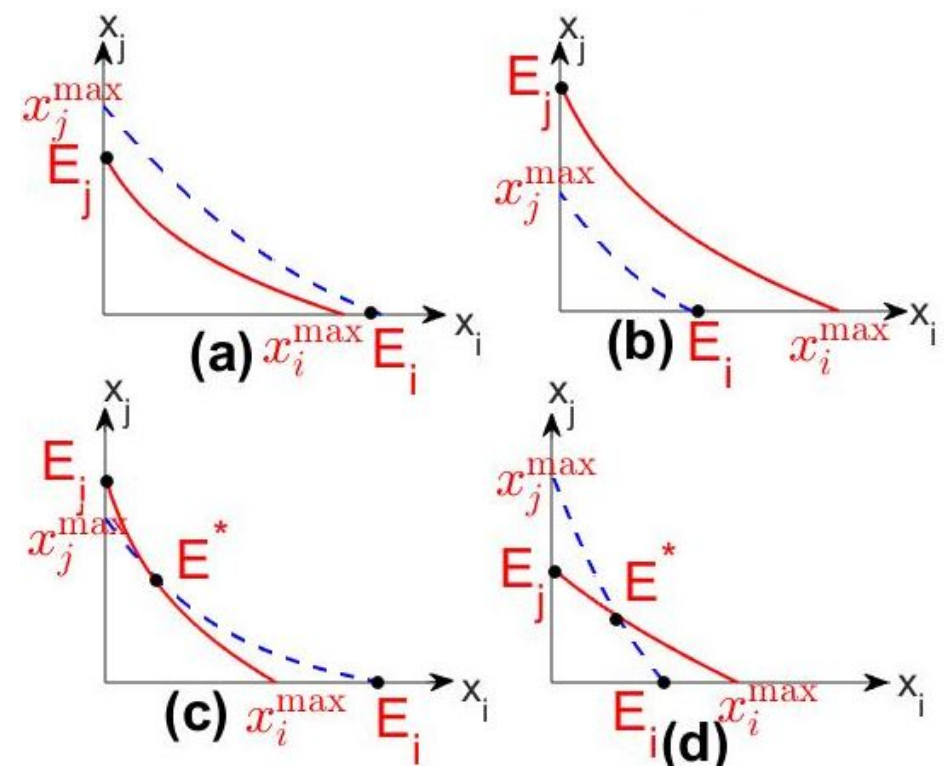

Figure 1: (a) and (b): none coexistence steady state, (c) and (d): one coexistence steady state $E^{*}$ according to resource and competition effects.
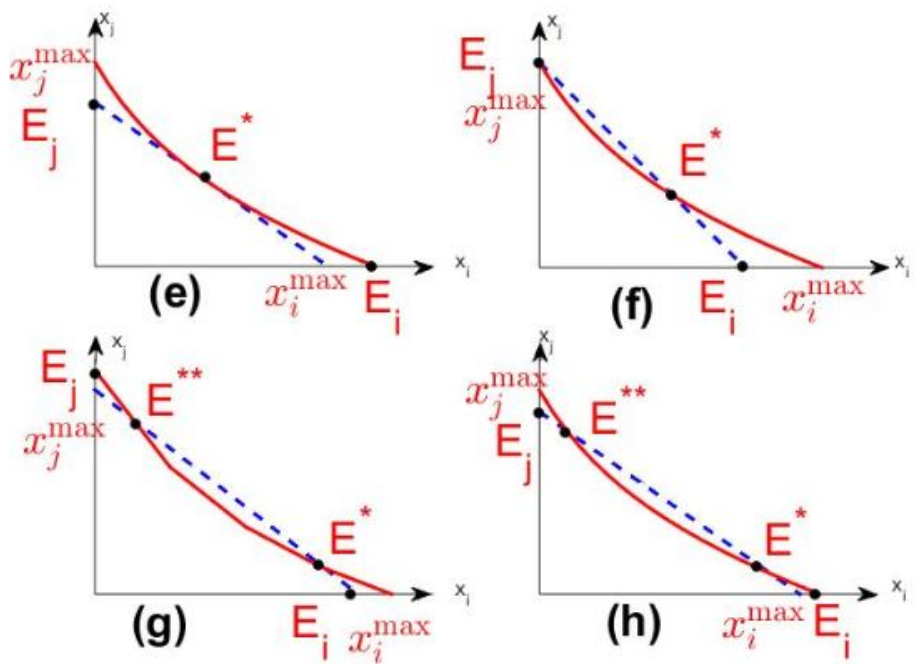

Figure 2: (e) and (f): one coexistence equilibrium $E^{*},(\mathrm{~g})$ and (h);: two coexistence equilibria $E^{*}$ and $E^{* *}$ according to resource and competition effects. 


\subsection{Local asymptotic stability of equilibria}

In this subsection, we study the local stability of model (2) equilibria.

\subsubsection{Exclusion of one species equilibria}

Proposition 3.1. Let $Z>0$. If $t_{i i} \leq t_{j i}$ then $E_{i}$ is LAS. Else, the following assertions holds:

(i) If $t_{i i}^{2} \geq t_{j i}\left(t_{i i}+\frac{s}{r} K\right)$ then $E_{i}$ is unstable;

(ii) If $t_{i i}^{2}<t_{j i}\left(t_{i i}+\frac{s}{r} K\right)$ then there exists $Z_{c_{i}}>0$ so that $E_{i}$ is LAS for the system (2) if $Z \geq Z_{c_{i}}$ else $E_{i}$ is unstable.

Moreover, when $E_{i}$ is unstable it holds that

- its unstable subspace: $\left(E_{i}^{u}\right): x_{j}=\beta(Z) x_{i}+\frac{\lambda_{i 2}-\lambda_{i 1}}{s_{i j\left(K_{i j}+1\right)}}$ where $\beta(Z)=-\frac{\lambda_{i 2}-\lambda_{i 1}}{s_{i j}\left(K_{i j}+1\right) K_{i i}}<0$, cross the domain $\Omega$

- and its stable subspace $\left(E_{i}^{s}\right)$ is the $\left(x_{i}-a x i s\right)$.

Proof. Let $Z>0$. Note that first, the eigenvalues of Jacobian matrix at $E_{i}$ Appendix (B.36) are $\lambda_{i 1}=-r_{i} Z<0$ and $\lambda_{i 2}=\frac{s_{j i} r_{i} K_{i i}}{s_{i i}\left(1+K_{i i}\right)}\left(Z_{c_{i}}-Z\right)$. So, $v=x_{i}(1,0)$ is a eigenvector associate to $\lambda_{i 1}<0$. It follows $\left(x_{i}-\right.$ axis $)$ is the stable subspace of the exclusion equilibrium $E_{i}$.

For the eigenvalue $\lambda_{i 2}$, if $t_{i i} \leq t_{j i}$ then $\lambda_{i 2}<0$. This implies $E_{i}$ is LAS for the system (2). Else, i.e, $t_{i i}>t_{j i}\left(Z_{c_{i}}>0\right)$, one can be pursuit the following additional hypotheses.

(i) If $t_{i i}^{2} \geq t_{j i}\left(t_{i i}+\frac{s}{r} K\right)$ then $\lambda_{i 2}>0$. By H. Grobman Theorem [39], $E_{i}$ is then unstable for the system (2). Moreover, it's clear that $\lambda_{i 2}>0$ provides the equation $\left(E_{i}^{u}\right)$ of the subspace unstable associated to $\lambda_{i 2}>0$.

(ii) It's obvious that if $t_{i i}^{2}<t_{j i}\left(t_{i i}+\frac{s}{r} K\right)$ then it remains the case $Z=Z_{c_{i}}>0$ to conclude for the hypothesis (ii).

Now, suppose that $Z=Z_{c_{i}}>0$. This implies $\lambda_{i 2}=0$. By setting $x_{i}=x_{i}-K_{i i}$ and $x_{j}=x_{j}$, it's clear that the origin is a equilibrium point of the following system: 


$$
\left\{\begin{array}{l}
\frac{d x_{i}}{d t}=\left(\frac{r_{i} Z}{1+x_{j}}\right)\left(x_{i}+K_{i i}\right)-s_{i i}\left(x_{i}+K_{i i}\right)^{2}-s_{i j}\left(x_{i}+K_{i i}\right) x_{j} \\
\frac{d x_{j}}{d t}=\left(\frac{r_{j} Z}{1+x_{i}+K_{i i}}\right) x_{j}-s_{j j} x_{j}^{2}-s_{j i}\left(x_{i}+K_{i i}\right) x_{j} .
\end{array}\right.
$$

From the jacobian matrix Appendix (B.39) of system (19) at origin, we give the two eigenvectors $v_{i}=(1,0), v_{j}=(-\gamma, 1)$, and the following matrix

$$
P^{-1}=\left(\begin{array}{ll}
1 & \gamma \\
0 & 1
\end{array}\right)
$$

Setting

$$
\left(\begin{array}{l}
X \\
Y
\end{array}\right)=P^{-1}\left(\begin{array}{l}
x_{i} \\
x_{j}
\end{array}\right)
$$

with $\gamma=\frac{s_{i j}}{s_{i i}}\left(K_{i j}+1\right)$, we obtain:

$$
\left\{\begin{aligned}
\dot{X}= & -2 r_{i} Z X-s_{i i} X^{2}+\psi_{1} Y+\psi_{2} Y^{2}+\psi_{3} X Y-s_{i i} K_{i i}^{2} \\
& +\frac{r_{i} Z\left(K_{i i}+X-\gamma Y\right)}{1+Y}+\frac{r_{j} Z \gamma Y}{1+K_{i i}+X-\gamma Y} \\
\dot{Y}= & -s_{j i} K_{i i} Y+\left(s_{j i} \gamma-s_{j j}\right) Y^{2}-s_{j i} X Y+\frac{r_{j} Z Y}{1+K_{i i}+X-\gamma Y}
\end{aligned}\right.
$$

where $X-\gamma Y=x_{i} \geq 0$,

$$
\begin{aligned}
& \psi_{1}=\left(2 s_{i i} \gamma-s_{i j}-s_{j i} \gamma\right) K_{i i}, \\
& \psi_{2}=\left(s_{i j}-s_{i i}-s_{j j}\right) \gamma \\
& \psi_{3}=2 s_{i i} \gamma-s_{i j}-s_{j i} \gamma .
\end{aligned}
$$

This system (22) can be rewritten in this useful form:

$$
\dot{V}=A V+Q(V)+o(\|(X, Y)\|)
$$

with $V=\left(\begin{array}{l}X \\ Y\end{array}\right), Q(V)=\left(\begin{array}{l}F(V) \\ G(V)\end{array}\right)$ where $A=\left(\begin{array}{cc}-r_{i} Z & \psi_{1} \\ 0 & -s_{j i} K_{i i}\end{array}\right)$,

$$
G(X, Y)=\left(s_{j i} \gamma-s_{j j}\right) Y^{2}-s_{j i} X Y
$$


and

$$
F(X, Y)=-s_{i i} X^{2}+\psi_{2} Y^{2}+\psi_{3} X Y
$$

with $Q(0,0)=(0,0)$ and $J Q(0,0)=\left(\begin{array}{ll}0 & 0 \\ 0 & 0\end{array}\right)$ where $J Q$ denotes the jacobian matrix of $Q$ Appendix (B.37).

Thus, since the matrix $A$ has negatives eigenvalues then $E_{i}$ is LAS for the system (2). This ends the proof.

Remark 3.3. Changing $i$ and $j$ roles in Proposition. 3.1, it can be obtain similarly results for the exclusion equilibrium state $E_{j}$ as a corollary.

These results will be useful for proving the global asymptotic stability of equilibria in section 4 .

\subsubsection{Coexistence equilibrium $E^{*}$}

Let $Z>0$ so that a coexistence equilibrium point $E^{*}$ exists, it derives that the trace and the determinant of the Jacobian matrix of the system (2) at $E^{*}$ (see Appendix (B.38)) can be expressed as

$$
\operatorname{Trace}\left(J\left(E^{*}\right)\right)=-\left(s_{i i} x_{i}^{*}+s_{j j} x_{j}^{*}\right)<0 .
$$

and

$$
\operatorname{det}\left(J\left(E^{*}\right)\right)=\frac{x_{i}^{*} x_{j}^{*}\left(1+x_{i}^{*}+x_{j}^{*}\right)}{\left(1+x_{i}^{*}\right)\left(1+x_{j}^{*}\right)}\left[\alpha_{c}-\alpha_{z}\left(E^{*}\right)\right]
$$

respectively, where

$$
\alpha_{c}=r_{i}^{\prime} r_{j}^{\prime}\left(t_{i i} t_{j j}-t_{i j} t_{j i}\right)
$$

and

$$
\alpha_{z}\left(E^{*}\right)=\frac{f\left(x_{i}^{*}, x_{j}^{*}\right)}{1+x_{i}^{*}+x_{j}^{*}}
$$

with

$$
\begin{aligned}
f\left(x_{i}^{*}, x_{j}^{*}\right) & =2 s_{i i} s_{j i}\left(x_{i}^{*}\right)^{2}+2 s_{j j} s_{i j}\left(x_{j}^{*}\right)^{2} \\
& +s_{j i}\left(s_{i i}+s_{i j}\right) x_{i}^{*}+s_{i j}\left(s_{j j}+s_{j i}\right) x_{j}^{*}+4 s_{i j} s_{j i} x_{i}^{*} x_{j}^{*} .
\end{aligned}
$$

These expressions (23), (24), (25) and (26) obviously allow to give the behavior of the system (2) neighborhood of $E^{*}$ in the following Proposition. 
Table 2: Notations of some Hypotheses.

\begin{tabular}{lc}
\hline Notations & Conditions \\
\hline$B_{i}$ & $t_{i i} \leq t_{j i}$ \\
$B_{j}$ & $t_{j j} \leq t_{i j}$ \\
$A_{i}$ & $t_{i i}^{2}<t_{j i}\left(t_{i i}+\frac{s}{r} K\right)$ \\
$A_{j}$ & $t_{j j}^{2}<t_{i j}\left(t_{j j}+\frac{s}{r} K\right)$ \\
$\hat{Z}$ & $\max _{k \in\{i, j\}}\left\{Z_{c_{k}}\right\}$ \\
$\check{Z}$ & $\min _{k \in\{i, j\}}\left\{Z_{c_{k}}\right\}$ \\
\hline
\end{tabular}

Proposition 3.2 (Coexistence of species). Let $Z>0$ so that a coexistence equilibrium point $E^{*}$ exists. Then, the following assertions hold for the system (2).

(i) If $\alpha_{c} \leq 0$ then $E^{*}$ is unstable.

(ii) For any $\alpha_{c}>0$, if $\alpha_{z}\left(E^{*}\right)<\alpha_{c}$ then $E^{*}$ is LAS. Else, if $\alpha_{z}\left(E^{*}\right)=\alpha_{c}$ then $E^{*}$ is stable, else $E^{*}$ is unstable.

(iii) if $E^{*}$ is unstable then it admits a stable manifold lying in the interior of $\Omega$.

For simplicity, we will use the notations of conditions given in table 2 in the following sections.

\section{Asymptotic Behaviors: Resource Based Model Outcome}

In this section, we consider that $Z>0$ and determine the local and global asymptotic stability of system (2) equilibria that exists. For this purpose, we will use the following auxiliary function

$$
g:\left[0, \min \left(K_{j j}, x_{j}^{\max }\right)\right] \rightarrow \mathbb{R}
$$

defined by

$$
g(x)=g_{i}(x)-g_{j}(x)
$$




\subsection{Balanced intra-inter specific competition effects}

In this subsection, we assume that the condition (5) is satisfied, i.e., $t_{i i} t_{j j}=t_{i j} t_{j i}$. This condition is equivalent to $\alpha_{c}=0$ (cf. (25)) and implies that the condition $\bar{B}_{i}$ is equivalent to $B_{j}$. In addition, the system (11) becomes

$$
\left\{\begin{array}{l}
a_{2}\left(x_{i}^{*}\right)^{2}+a_{1} x_{i}^{*}+a_{0}=0, \\
\frac{r_{j} Z}{s_{j j}\left(1+x_{i}^{*}\right)}-\frac{s_{j i}}{s_{j j}} x_{i}^{*}=x_{j}^{*},
\end{array}\right.
$$

where $a_{2}=e_{i} Z>0, a_{1}=\left(e_{i}+d_{i}\right) Z$ and $a_{0}=s_{i j} r_{j}^{2}\left(Z_{c_{i}}-Z\right) Z$ with $d_{i}=$ $s_{i j} r_{j}^{2} Z_{c_{i}}$.

Therefore, the $x_{i}^{*}$-Equation in (28) admits at most one positive root given by

$$
x_{i}^{*}=\frac{-a_{1}+\sqrt{a_{1}^{2}-4 a_{2} a_{0}}}{2 a_{2}} \text { if and only if } a_{0}<0 .
$$

This equivalence will be useful in the proof of following theorems.

Theorem 4.1. Suppose that the conditions $\bar{B}_{i}$ and $A_{i}$ are satisfied. Then for the system (2), there exists a bifurcation value $\hat{Z}>0$ of the resource $Z$ such that:

(i) for any $Z>\hat{Z}$, there exists a unique coexistence equilibrium $E^{*}$ which is unstable with the stable manifold is in interior of $\Omega$ and the two exclusion of one species equilibria $E_{i}$ and $E_{j}$ are both LAS.

(ii) for any $Z \leq \hat{Z}$, there is no coexistence equilibrium, $E_{i}$ is unstable and $E_{j}$ is $L A S$.

Proof. Let $\bar{B}_{i}$ and $A_{i}$ are satisfied. Notice that from $\bar{B}_{i}$ we have $Z_{c_{i}}>0$ and $B_{j}$. Furthermore, $A_{i}$ implies $Z_{c_{i}}<Z_{\max }$.

(i) Suppose that $Z>Z_{c_{i}}$. This means $a_{0}<0$, then there exists at most one $E^{*}$. From $\bar{B}_{i}$ and (27) it holds that $g(0)=K_{i i}-x_{i}^{\max }>0$. But, by calculation, we have both $g\left(x_{j}^{\max }\right)=-g_{j}\left(x_{j}^{\max }\right)<0$ and $B_{j}$ implies that $x_{j}^{\max } \leq K_{j j}$. In addition, according to $g \quad:\left[0 ; x_{j}^{\max }\right] \rightarrow \mathbb{R}$ is continuous, there exists $\left.x_{j}^{*} \in\right] 0, x_{j}^{\max }\left[\right.$ such that $g\left(x_{j}^{*}\right)=0$ when $Z>Z_{c_{i}}$. Thus, $E^{*}$ exists and is unique. The results in Lemma 3.3 and 3.4 complete the proof of this assertion $(i)$.

(ii) If $Z \leq Z_{c_{i}}$ then $a_{0} \geq 0$. Therefore, there exists no coexistence equilibrium $E^{*}$ in $\Omega$. This completes the proof. 
Table 3: Existence and local stability of steady states according to $B_{i}, B_{j}, A_{i}, \hat{Z}$ and $\check{Z}$ are defined in the table 2 .

\begin{tabular}{lccc}
\hline Conditions & $E_{i}$ & $E_{j}$ & $E^{*}$ \\
\hline $\bar{B}_{i} A_{i}(Z<\hat{Z})$ & $\mathrm{U}$ & LAS & \\
$\bar{B}_{i} A_{i}(Z=\hat{Z})$ & LAS & LAS & \\
$\bar{B}_{i} A_{i}(Z>\hat{Z})$ & LAS & LAS & U \\
$\bar{B}_{i} \bar{A}_{i}$ & $\mathrm{U}$ & LAS & \\
\hline
\end{tabular}

Table 4: Existence and local stability of steady states according to $B_{i}, B_{j}, A_{i}, \hat{Z}$ and $\check{Z}$ are defined in the table 2 .

\begin{tabular}{lccc}
\hline Conditions & $E_{i}$ & $E_{j}$ & $E^{*}$ \\
\hline$B_{j} A_{i}(Z<\hat{Z})$ & U & LAS & \\
$B_{j} A_{i}(Z=\hat{Z})$ & LAS & LAS & \\
$B_{j} A_{i}(Z>\hat{Z})$ & LAS & LAS & U \\
$B_{j} \bar{A}_{i}$ & U & LAS & \\
\hline
\end{tabular}

Table 5: Existence and local stability of steady states according to $B_{i}, B_{j}, A_{j}, \hat{Z}$ and $\check{Z}$ are defined in table 2 .

\begin{tabular}{lccc}
\hline Conditions & $E_{i}$ & $E_{j}$ & $E^{*}$ \\
\hline$B_{i} A_{j}(Z<\hat{Z})$ & LAS & U & \\
$B_{i} A_{j}(Z=\hat{Z})$ & LAS & LAS & \\
$B_{i} A_{j}(Z>\hat{Z})$ & LAS & LAS & U \\
$B_{i} \bar{A}_{j}$ & LAS & U & \\
\hline
\end{tabular}

Table 6: Existence and local stability of steady states according to $B_{i}, B_{j}, A_{j}, \hat{Z}$ and $\check{Z}$ are defined in table 2 .

\begin{tabular}{lccc}
\hline Conditions & $E_{i}$ & $E_{j}$ & $E^{*}$ \\
\hline $\bar{B}_{j} A_{j}(Z<\hat{Z})$ & LAS & U & \\
$\bar{B}_{j} A_{j}(Z=\hat{Z})$ & LAS & LAS & \\
$\bar{B}_{j} A_{j}(Z>\hat{Z})$ & LAS & LAS & U \\
$\bar{B}_{j} \bar{A}_{j}$ & LAS & U & \\
\hline
\end{tabular}


Therefore, from local asymptotic stability of equilibria states of system (2) summarized in tables 3 to 6 , it holds that balanced competition effects condition gives the CEP.

Lemma 4.1. Suppose that $\frac{1}{2} s_{j j}<s_{i j}$ satisfying $s_{j j} \neq s_{i j}$. Then

$$
W^{s}\left(E_{i}\right) \backslash\left\{E_{i}\right\} \subset \mathbb{R}^{2} \backslash \Omega
$$

where $W^{s}\left(E_{i}\right)$ denotes the stable manifold at $E_{i}$.

Proof. To determine the stable manifold from Taylor expansion of the system (19), we consider: $x_{j}=h\left(x_{i}\right)$ where $h\left(x_{i}\right)=Q_{3}\left(x_{i}\right)+o\left(x_{i}^{4}\right)$, with $Q_{3}\left(x_{i}\right)=a x_{i}^{2}+b x_{i}^{3}$. Thus, from $Q_{3}^{\prime}\left(x_{i}\right)=2 a x_{i}+3 b x_{i}^{2}$

$$
\begin{aligned}
f\left(x_{i}, Q_{3}\left(x_{i}\right)\right) & =\frac{r_{i} Z}{1+a x_{i}^{2}+b x_{i}^{3}}\left(x_{i}+K_{i i}\right) \\
& -s_{i i}\left(x_{i}+K_{i i}\right)^{2}-s_{i j}\left(x_{i}+K_{i i}\right)\left(a x_{i}^{2}+b x_{i}^{3}\right), \\
g\left(x_{i}, Q_{3}\left(x_{i}\right)\right) & =\frac{r_{j} Z}{1+x_{i}+K_{i i}}\left(a x_{i}^{2}+b x_{i}^{3}\right) \\
& -s_{j j}\left(a x_{i}^{2}+b x_{i}^{3}\right)^{2}-s_{j i}\left(x_{i}+K_{i i}\right)\left(a x_{i}^{2}+b x_{i}^{3}\right),
\end{aligned}
$$

the manifold invariance:

$$
Q_{3}^{\prime}\left(x_{i}\right) f\left(x_{i}, Q_{3}\left(x_{i}\right)\right)=g\left(x_{i}, Q_{3}\left(x_{i}\right)\right),
$$

and the hypothesis $\left.s_{j j} \in\right] 0,2 s_{i j} \backslash \backslash\left\{s_{i j}\right\}$, it consecutively holds

$$
-3 s_{i j} b^{3}=-3 s_{j j} b^{3}, b^{3}\left(s_{j j}-s_{i j}\right)=0 \Longrightarrow b=0
$$

and

$$
a^{2}\left(s_{j j} a-2 s_{i j} a-2 s_{i j} K_{i i}\right)=0 \Longrightarrow a=\frac{2 s_{i j} K_{i i}}{s_{j j}-2 s_{i j}}<0 .
$$

Thereafter, we obtain the stable manifold at $E_{i}$ :

$$
\left(W^{s}\left(E_{i}\right)\right): x_{j}=a x_{i}^{2} \quad(a<0)
$$

is outside of $\Omega \backslash\left\{E_{i}\right\}$ when $\left.s_{j j} \in\right] 0,2 s_{i j}\left[\backslash\left\{s_{i j}\right\}\right.$. This ends the proof.

Theorem 4.2 (CEP). Suppose $\bar{B}_{i}, A_{i}$ and the assumption of Lemma 4.1 holds. Then there exists a bifurcation value $\check{Z}>0$ such that: 
(i) for any $Z<\check{Z}, E_{j}$ is $G A S$ in $\Omega \backslash\left(x_{i}-\right.$ axis) for the system (2);

(ii) for any $Z \geq \check{Z}$, the coexistence equilibrium $E^{*}$ is unstable and the two exclusion equilibria $E_{i}$ and $E_{j}$ are both LAS for the system (2).

Proof. (i) Let $x_{0}=\left(x_{0 i}, x_{0 j}\right)$ be an initial condition inside of $\Omega$. The orbit $\theta^{+}\left(x_{0}\right)$ is positively bounded, since the domain $\Omega$ is positively invariant. Thanks to the Poincaré-Bendixson type trichotomy [36], the $\omega$ - limit of $x_{0}$ contains either:

$\left.i_{1}\right)$ an equilibrium point or

$i_{2}$ ) an closed orbit or

$\left.i_{3}\right)$ an equilibrium point and a homoclinic orbit of that point or a chain of unstable points.

If $\bar{B}_{i}, A_{i}$ and $Z<Z_{c_{i}}$ then the system (2) admits three hyperbolic points $E_{0}, E_{i}$ and $E_{j}$. In one hand, applying the Lemma 3.2, it follows that the system has no periodical orbit. The statement $i_{2}$ ) is not then satisfied.

Otherwise, the Lemma 4.1 according $\omega\left(x_{0}\right) \neq\left\{E_{i}\right\}$. Indeed, $E_{i}$ is a hyperbolic point thus, by Theorem (Butler-McGehee) in [38] there exists $z \neq E_{i}$ such as $z \in \omega\left(x_{0}\right)$ which is absurd because $\omega\left(x_{0}\right) \subset \Omega$. It yields that $E_{i}$ is not admissible to be a $\omega$ - limit set of an initial condition belonging to $\Omega$. In other terms, there is no chain of equilibrium points and $E_{i}$ has no homoclinic orbit. Besides,$E_{j}$ has no homoclinic orbit since it is LAS. The statement $\left.i_{3}\right)$ is not satisfied. Consequently $\omega\left(x_{0}\right)=\left\{E_{j}\right\}$.

We have shown that the $\omega$-limit set of $x_{0}$ contains only $E_{j}$ equilibrium which is attractive. We conclude that $E_{j}$ equilibrium is GAS in $\Omega \backslash\left(x_{i}-\right.$ axis) for the system (2).

The statement $i_{2}$ ) has been verified in the Proof of the Proposition 3.2. This completes this proof.

Remark 4.1. Under balanced competition effects condition, only the CEP is valid for the model (2) in two ways. Indeed, in lowest levels of resource, a exclusion of a species holds when its intra-specific competition effects is greater than its inter-specific competition effects on the other competing species. In sufficient level of resource, the exclusion of one species depend on two species initial densities. 


\subsection{Unbalanced intra-inter specific competition effects}

Throughout this subsection, we assume that $t_{i i} t_{j j}-t_{i j} t_{j i}>0$, this is equivalent to $\alpha_{c}>0$ (cf. (25)). Then, the density $x_{i}^{*}$ of species $i$ at a coexistence equilibrium (when it exists) satisfies the equation $P\left(x_{i}^{*}\right)=0$ where

$$
P\left(x_{i}^{*}\right)=\alpha_{c}\left(x_{i}^{*}\right)^{4}+a_{3}\left(x_{i}^{*}\right)^{3}+a_{2}\left(x_{i}^{*}\right)^{2}+a_{1} x_{i}^{*}+a_{0}
$$

Theorem 4.3. Suppose $\bar{B}_{i}, \bar{B}_{j}, A_{i}$ and $A_{j}$ holds. Then for the system (2) there exist two bifurcation values, $\check{Z}$ and $\hat{Z}$, of the resource $Z$ that are both positives such that:

(i) for any $Z \leq \check{Z}$ or $Z \geq \hat{Z}$, the system (2) admits a unique coexistence equilibrium in interior of $\Omega$.

(ii) for any $\check{Z}<Z<\hat{Z}$, the system (2) admits at most two coexistence equilibria in the interior of $\Omega$.

Moreover, the local asymptotic behavior of $E^{*}$ or $E^{* *}$ is given by assertion (ii) in Proposition 3.2.

Proof. Notice that from $\bar{B}_{i}$ and $\bar{B}_{j}$, we have $Z_{c_{i}}$ and $Z_{c_{j}}$ are both positive. Furthermore, $A_{i}$ and $A_{j}$ implies $Z_{c_{i}}<Z_{\max }$ and $Z_{c_{j}}<Z_{\max }$ respectively.

(i) Suppose that $Z<\check{Z}$. This means $K_{k k}<x_{k}^{\max }, \forall k \in\{i, j\}$ which implies:

$$
g(0)=g_{i}(0)-g_{j}(0)=K_{i i}-x_{i}^{\max }<0 .
$$

But, $g\left(K_{j j}\right)=g_{i}\left(K_{j j}\right)-g_{j}\left(K_{j j}\right)=g_{i}\left(K_{j j}\right)>0$. Then, from $g:\left[0, K_{j j}\right] \longrightarrow$ $\mathbb{R}$ is continuous so that $g(0) g\left(K_{j j}\right)<0$, there exists $\left.x_{j}^{*} \in\right] 0, K_{j j}[$ such as $g\left(x_{j}^{*}\right)=0$. Consider $\left.x_{j}^{*^{\prime}} \in\right] x_{j}^{*}, K_{j j}\left[\right.$ such as $g\left(x_{j}^{*^{\prime}}\right)=0$. Then, there exists $\epsilon>0$ so that $g\left(x_{j}^{*^{\prime}}-\epsilon\right)>0$ and $g\left(x_{j}^{*^{\prime}}+\epsilon\right)<0$. Therefore, by the intermediate value theorem, there exists $\left.x_{j}^{*^{\prime \prime}} \in\right] x_{j}^{*^{\prime}}+\epsilon, K_{j j}$ [ satisfying $g\left(x_{j}\right)=0$. So, from $a_{0} / a_{4}>0$, the polynomial $P(x)(12)$ admits fourth positive roots. Using Descartes criteria (see Appendix C), this obvious contradicts sign of $P(x)$ coefficients when $Z<\check{Z}$. Thus, the system (2) admits an unique equilibrium when $Z<\check{Z}$.

In the same manner, contradiction of $P^{\prime}(x)$ (13) coefficients sign holds for any $Z>\hat{Z}$. Thus $E^{*}$ exists and is unique for the system (2) for $Z<\check{Z}$ or $Z>\hat{Z}$. The results in Lemma 3.3 and Lemma 3.4 complete this proof of assertion $(i)$. 
Table 7: Existence and local stability of steady states according to $B_{i}, A_{i}, \check{Z}=Z_{c_{j}}$ and $\hat{Z}=Z_{c_{i}}$ which are defined in the table 2 when $\alpha_{c}<\alpha_{z}\left(E^{*}\right)$ and $\alpha_{c}<\alpha_{z}\left(E^{* *}\right)$.

\begin{tabular}{lcccc}
\hline Conditions & $E_{i}$ & $E_{j}$ & $E^{*}$ & $E^{* *}$ \\
\hline $\bar{B}_{i}(\check{Z}<0<Z<\hat{Z})$ & $\mathrm{U}$ & $\mathrm{LAS}$ & & \\
$\bar{B}_{i} A_{i}(\check{Z}<0<\hat{Z} \leq Z)$ & LAS & LAS & $\mathrm{U}$ & \\
$\bar{B}_{i}(0<Z<\check{Z})$ & $\mathrm{U}$ & $\mathrm{U}$ & $\mathrm{U}$ & \\
$\bar{B}_{i}(0<Z=\check{Z})$ & $\mathrm{U}$ & LAS & $\mathrm{U}$ & \\
$\bar{B}_{i} A_{i}(0<\check{Z}<Z<\hat{Z})$ & $\mathrm{U}$ & LAS & $\mathrm{U}$ & $\mathrm{U}$ \\
\hline
\end{tabular}

Table 8: Existence and local stability of steady states according to $B_{i}, A_{i}, \check{Z}=Z_{c_{j}}$ and $\hat{Z}=Z_{c_{i}}$ which are defined in table 2 when $\alpha_{c}>\alpha_{z}\left(E^{*}\right)$ and $\alpha_{c}>\alpha_{z}\left(E^{* *}\right)$.

\begin{tabular}{lcccc}
\hline Conditions & $E_{i}$ & $E_{j}$ & $E^{*}$ & $E^{* *}$ \\
\hline $\bar{B}_{i}(\check{Z}<0<Z<\hat{Z})$ & U & LAS & & \\
$\bar{B}_{i} A_{i}(\check{Z}<0<\hat{Z} \leq Z)$ & LAS & LAS & LAS & \\
$\bar{B}_{i}(0<Z<\check{Z})$ & U & U & LAS & \\
$\bar{B}_{i}(0<Z=\check{Z})$ & U & LAS & LAS & \\
$\bar{B}_{i} A_{i}(0<\check{Z}<Z<\hat{Z})$ & U & LAS & LAS & LAS \\
\hline
\end{tabular}

(ii) Without loss generality, set $\check{Z}=Z_{c_{i}}$ and suppose that $Z_{c_{i}}<Z<$ $Z_{c_{j}}$. This means $x_{j}^{\max }-K_{j j}<0$ and $x_{i}^{\max }-K_{i i}>0$. By calculation, $g(0)=K_{i i}-x_{i}^{\max }<0$ and $g\left(x_{j}^{\max }\right)=-g_{j}\left(x_{j}^{\max }\right)<0$. If for any $\left.\gamma \in\right] 0 ; x_{j}^{\max }[$ $g(\gamma)<0$, then there is no coexistence equilibrium for the system (2).

Else, suppose that there exists $\left.\gamma_{0} \in\right] 0 ; x_{j}^{\max }\left[/ g\left(\gamma_{0}\right)=0\right.$ and $g(\gamma)<$ $0, \forall \gamma \neq \gamma_{0}$. Then, the system (2) admits a unique coexistence equilibrium. Finally, when it exists $\left.\gamma_{1} \in\right] 0 ; x_{j}^{\max }\left[\right.$ so that $g\left(\gamma_{1}\right)>0$. Applying twice the intermediate value theorem, there exist two points $\left.x_{j}^{*} \in\right] 0, \gamma_{1}\left[\right.$ and $x_{j}^{* *} \in$ ]$\gamma_{1}, x_{j}^{\max }$ [ solutions of $g(\tau)=0$. Combining the necessary conditions in Lemma 3.3, relations between roots and Descartes criteria (see Appendix C) it follows that only $E^{*}$ and $E^{* *}$ exist for the system (2). This completes the proof of assertion $(i i)$.

Therefore, the conclusions on local asymptotic stability of equilibria of system (2) when unbalanced competition effects hypotheses is supposed, are summarized in tables 7 to 11 . 
Table 9: Existence and local stability of steady states according to conditions $B_{j}, A_{j}, \check{Z}=Z_{c_{i}}$ and $\hat{Z}=Z_{c_{j}}$ which are defined in table $2 \alpha_{c}<\alpha_{z}\left(E^{*}\right)$ and $\alpha_{c}<\alpha_{z}\left(E^{* *}\right)$.

\begin{tabular}{lcccc}
\hline Conditions & $E_{i}$ & $E_{j}$ & $E^{*}$ & $E^{* *}$ \\
\hline $\bar{B}_{j}(\check{Z}<0<Z<\hat{Z})$ & $\mathrm{LAS}$ & $\mathrm{U}$ & & \\
$\bar{B}_{j} A_{j}(\check{Z}<0<\hat{Z} \leq Z)$ & $\mathrm{LAS}$ & $\mathrm{LAS}$ & $\mathrm{U}$ & \\
$\bar{B}_{j}(0<Z<\check{Z})$ & $\mathrm{U}$ & $\mathrm{U}$ & $\mathrm{U}$ & \\
$\bar{B}_{j}(Z=\check{Z}>0)$ & $\mathrm{LAS}$ & $\mathrm{U}$ & $\mathrm{U}$ & \\
$\bar{B}_{j} A_{j}(0<\check{Z}<Z<\hat{Z})$ & $\mathrm{LAS}$ & $\mathrm{U}$ & $\mathrm{U}$ & $\mathrm{U}$ \\
$\bar{B}_{j} \bar{A}_{j}(\check{Z}<0<Z)$ & $\mathrm{LAS}$ & $\mathrm{U}$ & & \\
\hline
\end{tabular}

Table 10: Existence and local stability of steady states according to $B_{j}, A_{j}, \check{Z}=$ $Z_{c_{i}}$ and $\hat{Z}=Z_{c_{j}}$ which are defined in table $2 \alpha_{c}>\alpha_{z}\left(E^{*}\right)$ and $\alpha_{c}>\alpha_{z}\left(E^{* *}\right)$.

\begin{tabular}{lcccc}
\hline Conditions & $E_{i}$ & $E_{j}$ & $E^{*}$ & $E^{* *}$ \\
\hline $\bar{B}_{j}(\check{Z}<0<Z<\hat{Z})$ & LAS & U & & \\
$\bar{B}_{j} A_{j}(\check{Z}<0<\hat{Z} \leq Z)$ & LAS & LAS & LAS & \\
$\bar{B}_{j}(0<Z<\check{Z})$ & U & U & LAS & \\
$\bar{B}_{j}(Z=\check{Z}>0)$ & LAS & U & LAS & \\
$\bar{B}_{j} A_{j}(0<\check{Z}<Z<\hat{Z})$ & LAS & U & LAS & LAS \\
$\bar{B}_{j} \bar{A}_{j}(\check{Z}<0<Z)$ & LAS & U & & \\
\hline
\end{tabular}

Table 11: Existence and local stability of steady states according to $B_{j}, A_{j}, \check{Z}=$ $Z_{c_{i}}$ and $\hat{Z}=Z_{c_{j}}$ are defined in table $2 \alpha_{c}<\alpha_{z}\left(E^{*}\right)$ and $\alpha_{c}>\alpha_{z}\left(E^{* *}\right)$.

\begin{tabular}{lcccc}
\hline Conditions & $E_{i}$ & $E_{j}$ & $E^{*}$ & $E^{* *}$ \\
\hline $\bar{B}_{j}(\check{Z}<0<Z<\hat{Z})$ & LAS & $\mathrm{U}$ & & \\
$\bar{B}_{j} A_{j}(\check{Z}<0<\hat{Z} \leq Z)$ & $\mathrm{LAS}$ & $\mathrm{LAS}$ & $\mathrm{U}$ & \\
$\bar{B}_{j}(0<Z<\check{Z})$ & $\mathrm{U}$ & $\mathrm{U}$ & $\mathrm{U}$ & \\
$\bar{B}_{j}(Z=\check{Z}>0)$ & $\mathrm{LAS}$ & $\mathrm{U}$ & $\mathrm{U}$ & \\
$\bar{B}_{j} A_{j}(0<\check{Z}<Z<\hat{Z})$ & $\mathrm{LAS}$ & $\mathrm{U}$ & $\mathrm{U}$ & LAS \\
$\bar{B}_{j} \bar{A}_{j}(\check{Z}<0<Z)$ & $\mathrm{LAS}$ & $\mathrm{U}$ & & \\
\hline
\end{tabular}


Table 12: Some parameters and their values.

\begin{tabular}{cccc}
\hline Parameters & $r_{i}$ & $r_{j}$ & $K$ \\
Values & 0.04 & 0.03 & 1000 \\
\hline
\end{tabular}

The following theorem gives a result on global asymptotic stability of the coexistence equilibrium.

Theorem 4.4 (Paradox of phytoplankton). Suppose $\bar{B}_{i}, \bar{B}_{j}, \frac{1}{2} s_{l l}<s_{l k} \forall l \neq$ $k \in\{i, j\}$ and $Z<\check{Z}$ are satisfied. Then there exists a bifurcation value $\alpha_{c}$ such that:

(i) for any $\alpha_{z}\left(E^{*}\right)<\alpha_{c}$, the unique coexistence equilibrium $E^{*}$ is $G A S$ in $\Omega \backslash\left(x_{i}-\right.$ axis $) \cup\left(x_{j}-\right.$ axis $)$ for the system (2).

(ii) for any $\alpha_{z}\left(E^{*}\right)>\alpha_{c}$, although the coexistence equilibrium point is unstable, the system (2) is persistent.

Proof. Suppose $\bar{B}_{i}, \bar{B}_{j}$ and $Z<\check{Z}$ then the exclusion equilibria are unstable. Furthermore, $\frac{1}{2} s_{l l}<s_{l k}, \forall l \neq k \in\{i, j\}$ implies that the stable manifold at each exclusion equilibrium satisfies $W^{s}\left(E_{k}\right) \backslash\left\{E_{k}\right\} \subset \mathbb{R}^{2} \backslash \Omega$.

(i) In same manner that we shown in the Proof of Theorem 4.2 the $\omega$-limit set of any initial condition $x_{0}$ contains only $E^{*}$ equilibrium which is attractive. We conclude that $E^{*}$ equilibrium is GAS in $\Omega \backslash\left(x_{i}-\right.$ axis $) \cup$ $\left(x_{j}-a x i s\right)$ for the system (2). The statement of (ii) has been verified in the proof of the Proposition 3.2.

\section{Numerical simulations}

In this section, we present some numerical simulations of the model (2) that collaborate the theoretical results obtained in the previous sections. They concern numeric solutions of equations derived from the model (2) by using the software Matlab R2015a. The parameters values defined in the table 12 and those satisfying conditions and hypotheses are used to show graphical results.

Using the routine fsolve of Matlab in the program AbasinMacArthur2.m (available under request from the authors), the Figure 3 shows the attraction basin of each exclusion equilibrium point when condition of Balanced 


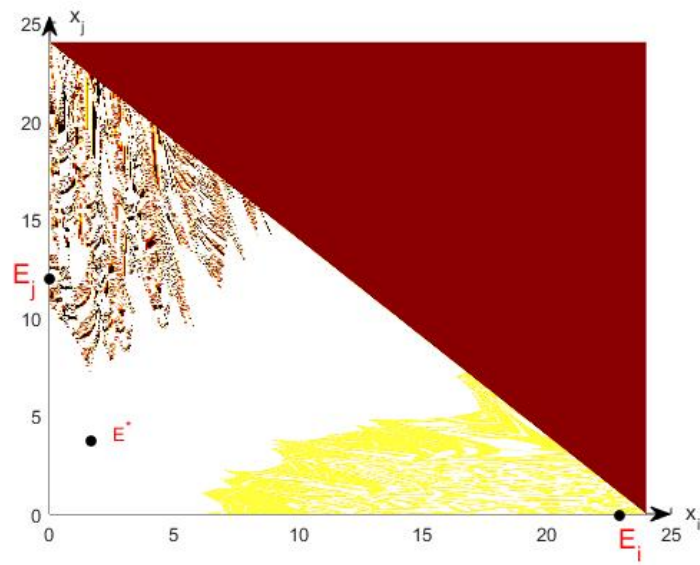

Figure 3: Illustration of the attraction basins of $E_{i}$ (yellow color) and $E_{j}$ (grey color) separated by the stable manifold of $E^{*}\left(W^{s}\left(E^{*}\right) \subset \Omega\right)$ corresponding to the case that $E_{i}$ and $E_{j}$ are LAS when $E^{*}$ is unstable for the model (2) with the parameters values $\alpha_{c}=0, s_{i i}=\frac{1}{3}, s_{j j}=\frac{1}{2}, s_{i j}=\frac{1}{3}, s_{j i}=\frac{1}{2}, Z=200$ and those in the table 12 .

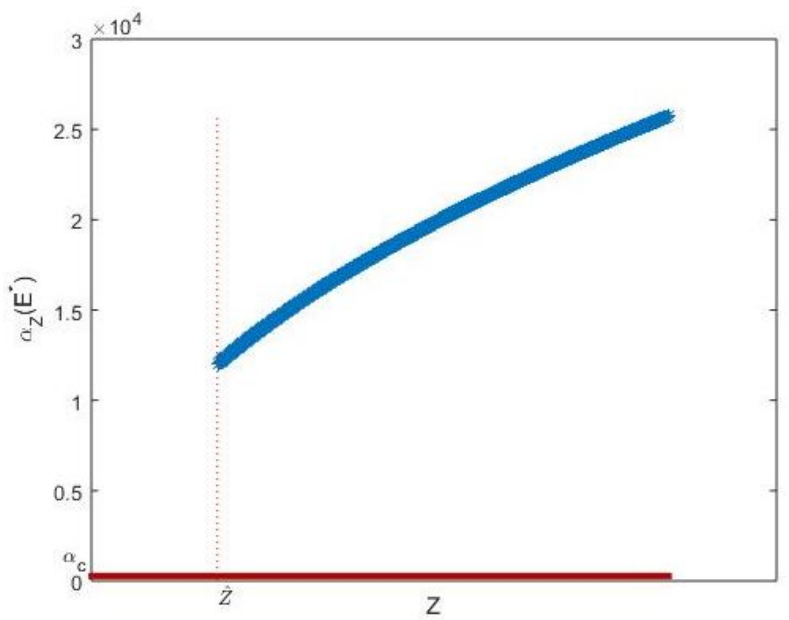

Figure 4: Illustration of the unique coexistence equilibrium is unstable under Balanced competition effects condition $\left(\alpha_{c}=0\right)$ when $t_{j j} \leq t_{i j}$ and $t_{i i}>t_{j i}$ by plotting the function $Z \mapsto \alpha_{Z}\left(E^{*}\right)$ (blue color)and $\alpha_{c}$ (red color) for $Z>\hat{Z}$ with the same parameters values which give Figure 3. 

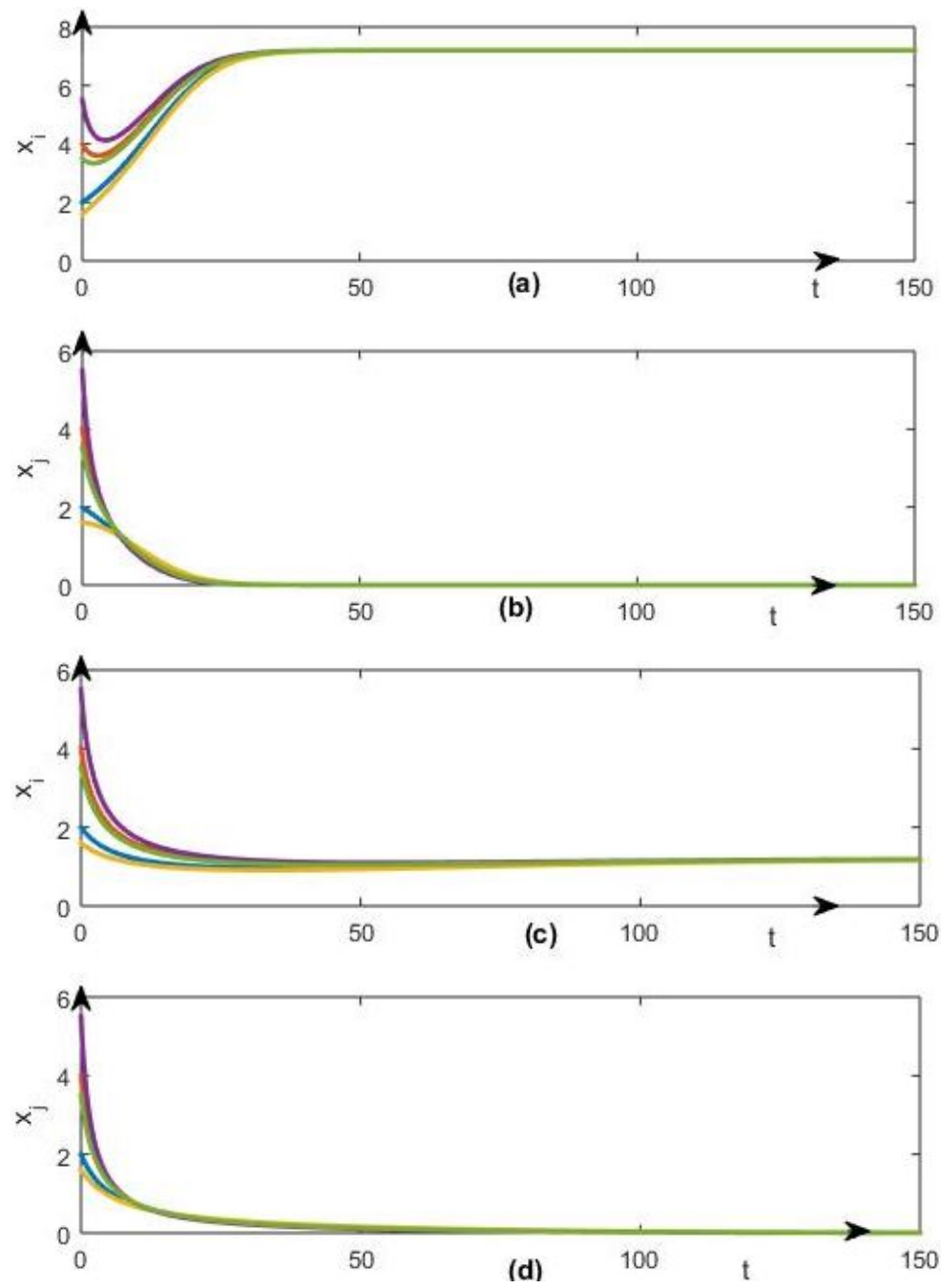

Figure 5: Populations $x_{i}$ and $x_{j}$ trajectories from initial conditions: $\left(x_{i 0}, x_{j 0}\right)=$ $(0.2,0.2) ;(0.4,0.4) ;(0.16,0.16) ;(0.55,0.55) ;(0.35,0.35)$ for $\alpha_{c}=0$ with parameters values given in Figure 3 and the resource $Z=1$ for (a), (b) and $Z=6$ for (c), (d).

Biomath Communications 8 (2021), 2112171, 10.11145/bmc.2021.12.171 29/44 

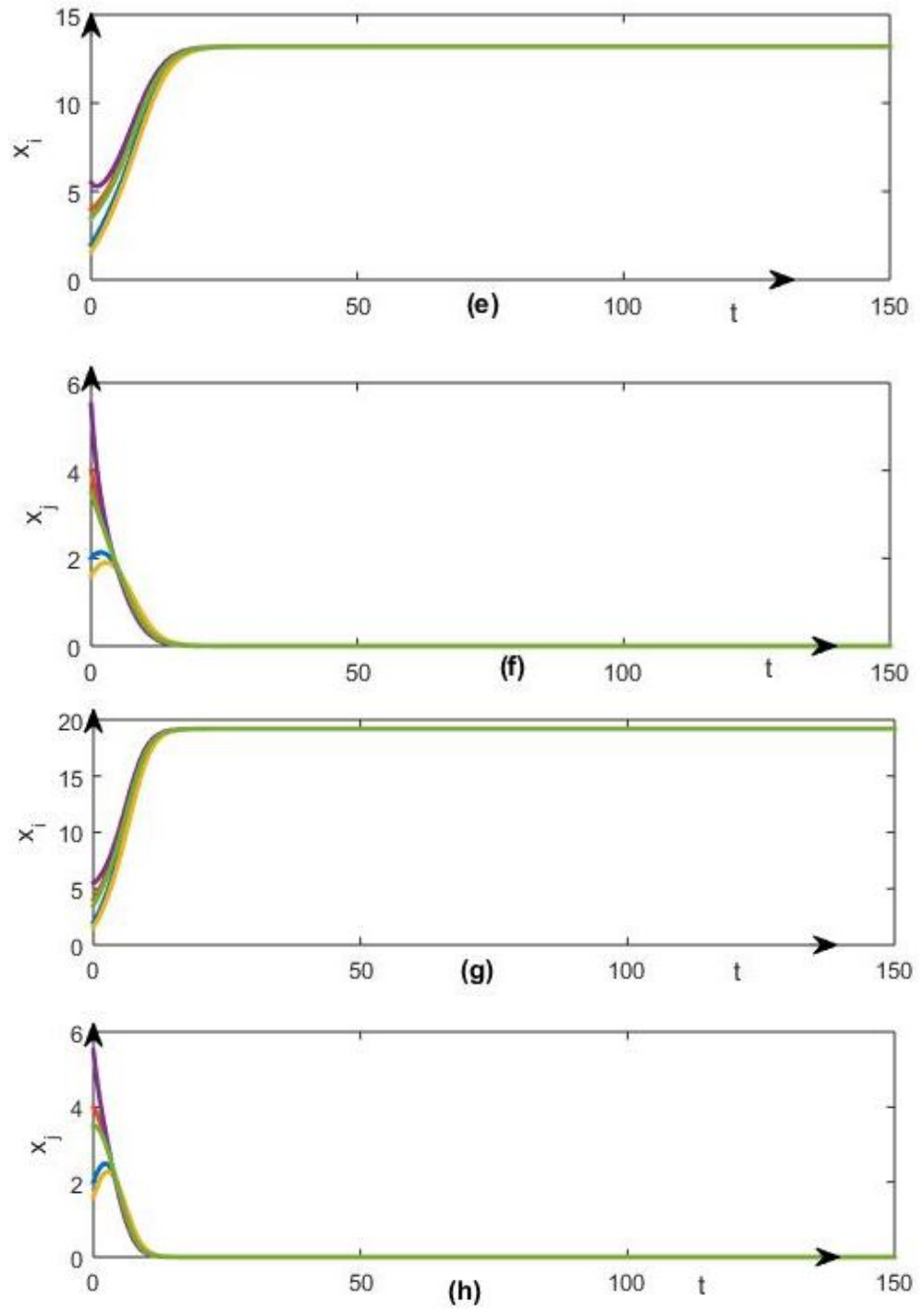

Figure 6: Populations $x_{i}$ and $x_{j}$ trajectories satisfying balanced competition effects $\left(\alpha_{c}=0\right)$ and the resource $Z=11$ for (e), (f) and $Z=16$ for (g),(h) with the same parameters values and initial conditions given in Figure 5. 
competition effects $\left(\alpha_{c}=0\right)$ is satisfied with $Z=200, t_{i i}=8.3333>$ $t_{j i}=16.6667, t_{j j}=16.6667>t_{i j}=8.3333$. These values provide $Z_{c_{i}}=$ $-4.1667<0, \hat{Z}=Z_{c_{j}}=16.6667$ as critic value of resource and the estimations of $E^{*}=(1.3609,3.7218), E_{i}=(24,0)$ and $E_{j}=(0,12)$ as unique coexistence, exclusion of species $j$ and exclusion of species $i$ equilibrium points respectively.

It can be seen in this figure (Figure 3 ) that the attraction basin of $E_{i}=(24,0)$ (yellow color) and the attraction basin of $E_{j}=(0,12)$ (grey color) are separated here by a surface (white color) of neither stable nor attractive points instead of the intending curve of the stable manifold at $E^{*}$. Clearly, this indicates that the system (2) converge towards to one exclusion equilibrium point as time goes to infinity according to the initial condition.

In addition, for these same parameters values, it can be seen in Figure 4 that the graph of the function $Z \longmapsto \alpha_{Z}$ (26) for $Z>16.6667$ is above the graph of the function $Z \longmapsto \alpha_{c}=0$ i.e the $Z$-axis. This confirms that the coexistence equilibrium $E^{*}=\left(x_{i}^{*}(Z), x_{j}^{*}(Z)\right)$ point exits and is unstable for $Z>16.6667$. Also, as it's depicted in Figure 5 and Figure 6, the system (2) converges to $E_{j}$ for many values of the resource $Z$ satisfying $Z<\hat{Z}$. Therefore, this confirms the model (2) suggests the CEP when the two species in competition for a common resource satisfy the balanced intra-inter specific competition effects condition as it states in Theorem 4.2.

Considering the case of unbalanced intra-inter specific competition effects when $\alpha_{c}=106940>0$ so that $t_{i i}=350.0000>t_{j i}=116.6667$, $t_{j j}=333.3333>t_{i j}=83.3333$. For $Z=800$, we obtain $E_{i}=(2.2857,0)$, $E_{j}=(0,2.4), \check{Z}=Z_{c_{i}}=70$ and $\hat{Z}=Z_{c_{j}}=1000$ by calculation and the numeric resolution of equations (11) gives the existence of the two coexistence equilibrium points estimated by $E^{*}=(1.7345,0.2706)$ and $E^{* *}=(1.2857,0.6000)$. From putting these approximate coordinates of each coexistence equilibrium point in equation (26), it derives $\alpha_{Z}\left(E^{*}\right)=$ $146.0917<\alpha_{c}$ and $\alpha_{Z}\left(E^{* *}\right)=113.3157<\alpha_{c}$. The evaluations at a long term in time towards infinity of the solution of equations (2) from any initial condition $x_{0} \in \Omega_{s}$ with $K_{s}=9.8$ falling in a small neighborhood of an equilibrium point are in Figure 7.

It can be seen only the attraction basins of equilibria $E_{j}=(0,2.4)$, $E^{*}=(1.7345,0.2706)$ and $E^{* *}=(1.2857,0.6000)$ as it is depicted in Figure 8. This shows that $E_{i}=(2.2857,0)$ is unstable as well as $E_{j}=(0,2.4)$, 


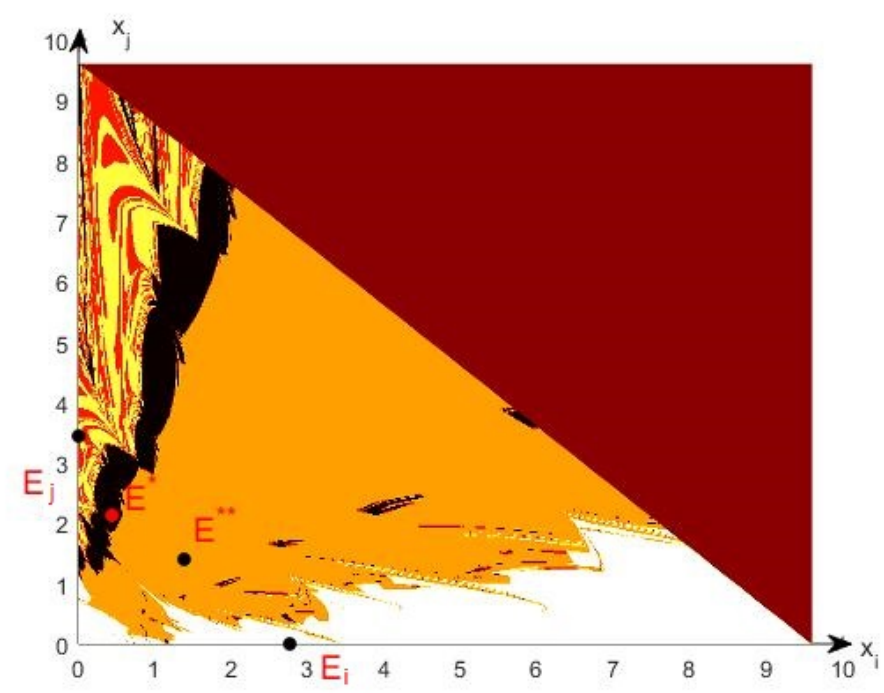

Figure 7: Illustration of the species $i$ exclusion by the attraction basins of $E_{j}$ (yelow), $E^{*}$ (black) and $E^{* *}$ (orange) with the parameters values $s_{i i}=14, s_{j j}=$ $10, s_{i j}=\frac{10}{3}, s_{j i}=\frac{7}{2}$, those in Table. 12 and for resource $Z=800$. It indicates that $E_{j}, E^{*}$ and $E^{* *}$ are LAS and $E_{i}$ (white color neighborhood) is unstable according to initial conditions $x_{0} \in \Omega_{s}$ with $K_{s}=8.9$.

$E^{*}=(1.7345,0.2706)$ and $E^{* *}=(1.2857,0.6000)$ are LAS. For $Z=1500>$ $\hat{Z}$, we obtain in the same manner that $E_{i}=(4.2857,0), E_{j}=(0,4.5)$ and $E^{*}=(0.7822,2.2511)$ are LAS as it's depicted in Figure 8 so that $\alpha_{Z}\left(E^{*}\right)=155.8808<\alpha_{c}$. Considering the case of unbalanced competition effects when $t_{i i}=300.0000>t_{j i}=100.0000$ and $t_{j j}=233.3333>t_{i j}=$ 58.3333 , that provide $\alpha_{c}=6.4167 e+04, \check{Z}=Z_{c_{i}}=600$ and $\hat{Z}=Z_{c_{j}}=$ 700. For $Z=500<\check{Z}$, it's obtained that $E_{i}=(1.6667,0)$ and $E_{j}=$ $(0,2.1429)$ are unstable and the unique coexistence equilibrium point $E^{*}=$ $(0.5627,1.1301)$ is LAS according to its attraction basin in Figure 9.

From the same parameters values with $Z$ varying, it is depicted in Figure 10 (a) the graphs of both functions $\left(Z \longmapsto\left\|E^{*}(Z)\right\|_{1}=x_{i}^{*}(Z)+x_{j}^{*}(Z)\right.$ and $\left.Z \longmapsto\left\|E^{* *}(Z)\right\|_{1}=x_{i}^{* *}(Z)+x_{j}^{* *}(Z)\right)$ that show the existence of zero, one or two coexistence equilibrium points. It can be seen in Figure 10 (b) the different positions between the graphs of the functions $Z \longmapsto \alpha_{Z}\left(E^{*}\right)$ (26) and $Z \longmapsto \alpha_{Z}\left(E^{* *}\right)$ (26) for $Z>0$ relatively to $Z \longmapsto \alpha_{c}>0$ that 


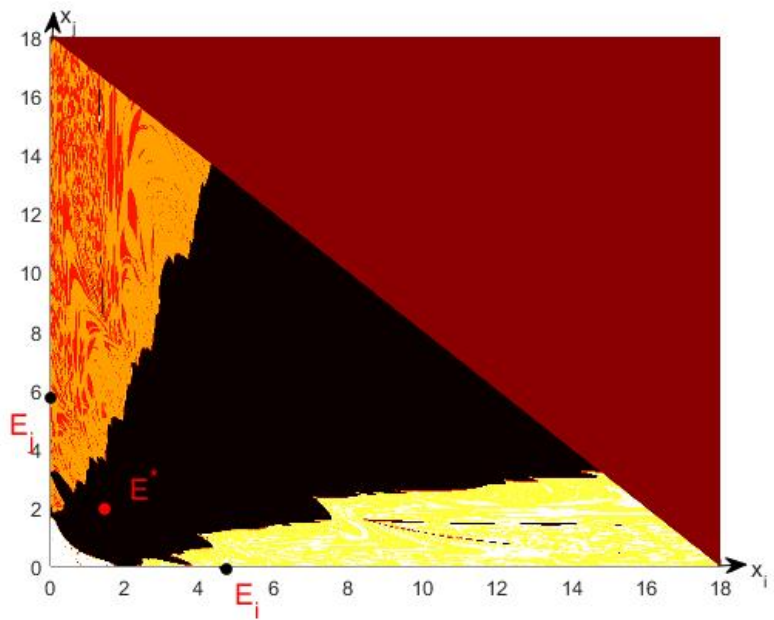

Figure 8: Illustration of the tri-stability of the system (2) concerning $E_{i}, E_{j}$ and the unique coexistence equilibrium $E^{*}$, obtained with same values parameters given in figure 7 and the resource value $Z=1500$. It shows species exclusion or coexistence according the initial $x_{0} \in \Omega_{s}$ with $K_{s}=18$.

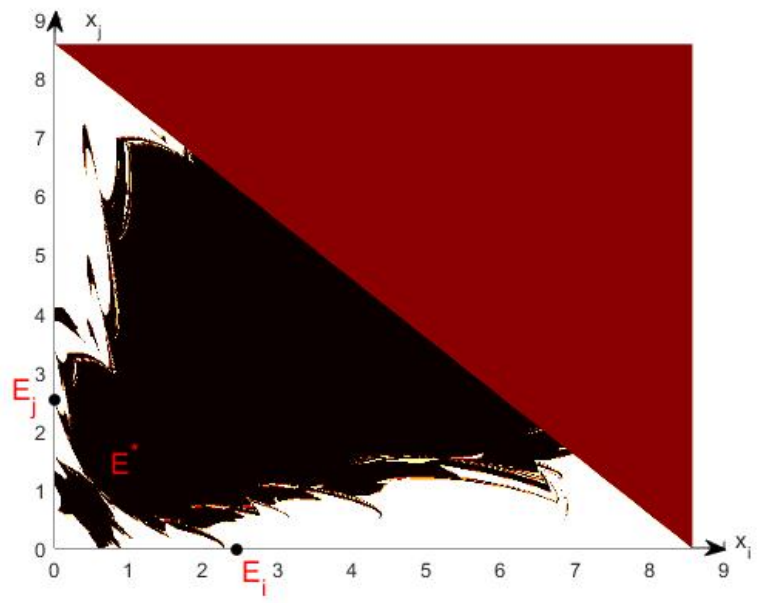

Figure 9: Illustration of assertion $(i)$ of the Theorem 4.4 when the two exclusion equilibria $E_{i}$ and $E_{j}$ are unstable and the unique coexistence equilibrium point $E^{*}$ is LAS, obtained with the parameters values $s_{i i}=12, s_{j j}=7, s_{i j}=\frac{7}{3}, s_{j i}=3$ and the resource value $Z=500$. It shows species coexistence according the initial $x_{0} \in \Omega_{s}$ with $K_{s}=8.9$. 


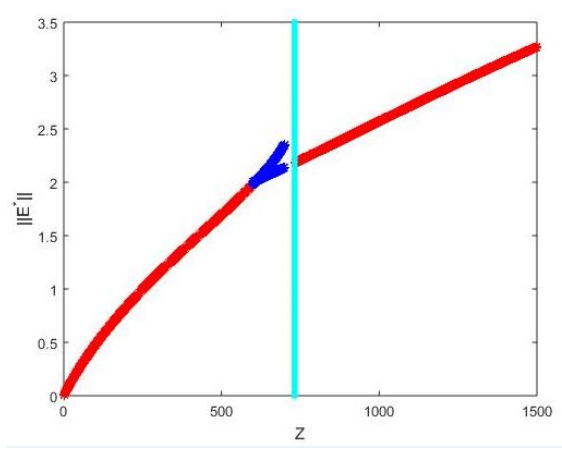

(a)

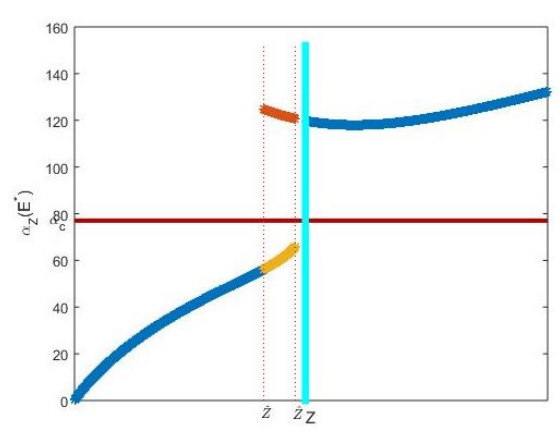

(b)

Figure 10: (a): Functions $\left(Z \longmapsto x_{i}^{*}(Z)+x_{j}^{*}(Z)\right)$ and $\left(Z \longmapsto x_{i}^{* *}(Z)+x_{j}^{* *}(Z)\right)$ graphs showing the existence of zero, one or two coexistence equilibrium points. (b) Graphic comparison of functions $Z \longmapsto \alpha_{Z}\left(E^{*}\right)$ (26) and $Z \longmapsto \alpha_{Z}\left(E^{* *}\right)$ (26) relatively to $Z \longmapsto \alpha_{c}>0$ given the existence of two coexistence equilibrium points with one is LAS and the other is unstable if $Z \in] \check{Z} ; \hat{Z}[=] 600 ; 700$ [ and. also an unique coexistence equilibrium point which is unstable if $Z \in] 0 ; 600$ [ and is LAS when $Z \in] 700+\epsilon ; 2000[(\epsilon>0)$.

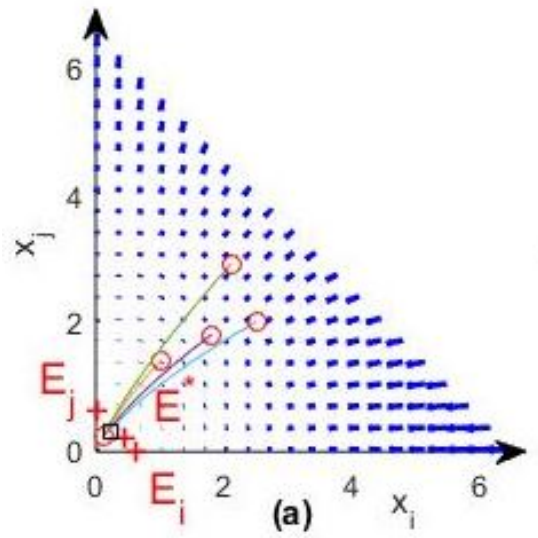

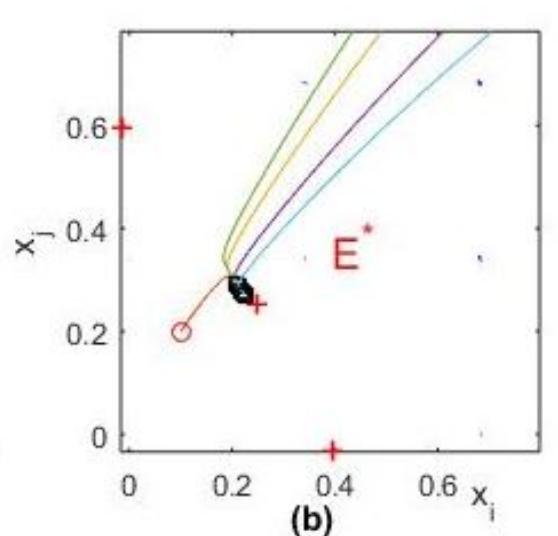

(b)

Figure 11: Illustration of the persistence of system (2) when any equilibrium point $\left(E_{i}, E_{j}\right.$ and $\left.E^{*}\right)$ is unstable with parameters values $s_{i i}=1, s_{j j}=$ $0.5, s_{i j}=\frac{1}{3}, s_{j i}=\frac{1}{2}$, the resource $Z=10$ and the initial conditions $(0.2,0.1) ;(1.4,1) ;(1.8,1.8) ;(2,2.1) ;(2.9,2.5)$. 
characterize the stability or the instability of coexistence equilibria points in the intervals $] 0 ; \check{Z}[$, $] \check{Z} ; \hat{Z}[$ and $] \hat{Z} ; Z_{\max }[$. These numeric results clearly indicate combination of statements in Proposition 3.2 and Theorem 4.3 as bifurcation diagrams. Considering the case of unbalanced competition effects when $t_{i i}=25.0000, t_{i j}=83.3333, t_{j j}=16.6667, t_{j i}=16.6667$ implies $0<\alpha_{c}=0.333<\alpha_{Z}=1.3028, E_{i}=(0.4,0), E_{j}=(0,0.6)$, $E^{*}=(0.2416,0.2416)$ and $Z_{\max }=83333, \check{Z}=Z_{c_{i}}=12.5000, Z=10<$ $Z_{c_{i}} \hat{Z}=Z_{c_{j}}=16.6667$, then $E_{i}, E_{j}$ and $E^{*}$ are unstable. This shows persistence species.

\section{Conclusion}

In this work, we have proposed and analyzed a new planar ordinary differential equations system as an extension of classical Lotka-Volterra competition model. This concerned the resource effects on two species coexistence or the exclusion of one of them when a permanent regime of resource availability and a density dependent resource acquirement for species are supposed. The mathematical analysis proved that the key factor of the competition outcome is the relation between intra and inter specific interference effects and sometimes the resource level importance. Indeed, it's shown that the Lotka-Volterra-MacArthur competition model can derived as a limit of the built model. The well known conclusions on Lotka-VolterraMacArthur competition model according to usual conditions (a)-(d) (see for instance diagne. et al) are not resource level dependent.

Thereafter, inspired by Lotka-Volterra underlining by Renshaw in [33], we introduced the hypotheses of balanced (5) and unbalanced (7)-(8) intra interspecific inferences from formulas that describe intra and inter specific competition effects between species relatively their rate of growth or rate of unit resource consummation growth.

The general analysis suggested the extinction of two species when there is non resource $Z$ in the system. Conversely, when the resource is permanent $Z>0$, it provided uniqueness and positivity of solution, a global attractor, properties that characterize a coexistence equilibrium point, the non existence of limit cycle by Dulac criteria and the LAS of exclusion and coexistence equilibria by Hartmann Grobman, local manifolds and center manifold theorems for the system (2). In addition, we proved that the CEP holds when two species satisfy the Balanced competition effects hypothesis 
whenever when it states the unbalanced competition effects condition, the model (2) goes to the CEP or the coexistence of species states according to the level of resource. In both conditions, it was exhibited that equilibrium points of the model (2) are all unstable. The Poincare-Bendixson Theorem [36] combined to model (2) obtained here properties is used to show one exclusion of species equilibrium GAS and coexistence of species equilibrium GAS.

The modifications of the classic prey-predator model of Lotka proposed in [31] as well as the epidemiological models proposed in [37] with polynomial factors can also be applied for this model.

Numerical simulations were perform to prove their congruence to theoretical results. In addition, it was established that the model can go to coexistence of species when equilibria are theoretically all unstable.

Finally, the presented model can be view as an important step towards a more thorough understanding of relationships between interference of species, resource dynamics and competition outcomes. Future work should include biogeography and variations of inference effects according to accessible resource dynamics that lead to a switched system or concerns resource ratio-based theory.

\section{A Proofs}

Lemma 3.3. From the system (11), one can have the following system of equations:

$$
\begin{aligned}
s_{i i} x_{i}^{*}\left(1+x_{j}^{*}\right) & =r_{i}^{\prime} Z-s_{i j} x_{j}^{*}\left(1+x_{j}^{*}\right), \\
x_{j}^{*} & =\frac{r_{j}^{\prime} Z-s_{j i} x_{i}^{*}\left(1+x_{i}^{*}\right)}{s_{j j}\left(1+x_{i}^{*}\right)} .
\end{aligned}
$$

From putting (A.30) in (A.29) and setting $\pi=r_{j}^{\prime} Z-s_{j i} x_{i}^{*}\left(1+x_{i}^{*}\right)$ and $\zeta=s_{j j}\left(1+x_{i}^{*}\right)$, it holds the following equation:

$$
\zeta^{2} r_{i}=(\zeta+\pi)\left(\zeta s_{i i} x_{i}^{*}+s_{i j} \pi\right)=\pi^{2}+s_{i i} x_{i}^{*} \zeta^{2}+\left(s_{i i} x_{i}^{*}+s_{i i}\right) \zeta \pi
$$


Therefore, from the following little algebra calculations of equation (A.31) terms

$$
\begin{aligned}
s_{i j} \pi^{2} & =s_{i j}\left(r_{j}^{2}+s_{j i}^{2}\left(x_{i}^{*}\right)^{2}\left(1+x_{i}^{*}\right)^{2}-2 r_{j} s_{j i} x_{i}^{*}\left(1+x_{i}^{*}\right)\right) \\
& =r_{j}^{2} s_{i j}-2 r_{j} s_{i j} s_{j i} x_{i}^{*}+s_{i j} s_{j i}^{2}\left(x_{i}^{*}\right)^{4} \\
& +s_{i j} s_{j i}\left(s_{j i}-2 r_{j}\right)\left(x_{i}^{*}\right)^{2}+2 s_{i j} s_{j i}^{2}\left(x_{i}^{*}\right)^{3}, \\
s_{i i} x_{i}^{*} \zeta^{2} & =s_{i i} x_{i}^{*}\left(s_{j j}^{2}+2 s_{j j}^{2} x_{i}^{*}+s_{j j}^{2}\left(x_{i}^{*}\right)^{2}\right) \\
& =s_{i i} s_{j j}^{2} x_{i}^{*}+2 s_{i i} s_{j j}^{2}\left(x_{i}^{*}\right)^{2}+s_{i i} s_{j j}^{2}\left(x_{i}^{*}\right)^{3}, \\
\zeta^{2} r_{i} & =r_{i} s_{j j}^{2}+2 r_{i} s_{j j}^{2} x_{i}^{*}+r_{i} s_{j j}^{2}\left(x_{i}^{*}\right)^{2}, \\
\Pi & =\left(s_{i i} x_{i}^{*}+s_{i j}\right) \zeta \pi \\
& =\left(s_{i i} x_{i}^{*}+s_{i j}\right)\left(r_{j} s_{j j}+s_{j j}\left(r_{j}-s_{j i}\right) x_{i}^{*}-2 s_{j j} s_{j i}\left(x_{i}^{*}\right)^{2}-s_{j j} s_{j i}\left(x_{i}^{*}\right)^{3}\right) \\
& =r_{j} s_{i j} s_{j j}+s_{j j}\left(r_{j}\left(s_{i j}+s_{i i}\right)-s_{i j} s_{j i}\right) x_{i}^{*} \\
& +s_{j j}\left(r_{j}\left(s_{i i}-s_{j i}\left(s_{i i}+2 s_{i j}\right)\right)\left(x_{i}^{*}\right)^{2}\right. \\
& +s_{j j} s_{j i}\left(s_{i j}-2 s_{i i}\right)\left(x_{i}^{*}\right)^{3}-s_{i i} s_{j j} s_{j i}\left(x_{i}^{*}\right)^{4},
\end{aligned}
$$

with $r_{k}=r_{k}^{\prime} Z(k=i, j)$, it derives the coefficients of the polynomial (12)

$$
\begin{aligned}
a_{4} & =s_{j i} \alpha_{c} \quad \text { with } \alpha_{c}=s_{i i} s_{j j}-s_{j i} s_{i j}, \\
a_{3} & =\left(2 s_{j i}-s_{j j}\right) \alpha_{c}, \\
a_{2} & =e_{i} Z+\left(-2 s_{j j}-r_{j}^{\prime} Z+s_{j i}\right) \alpha_{c} \text { where } e_{i}=s_{j j}^{2} r_{i}^{\prime}+s_{j i} s_{i j} r_{j}^{\prime}>0, \\
a_{1} & =e_{i} Z-\left(s_{j j}+r_{j}^{\prime} Z\right) \alpha_{c}+d_{i} Z \\
& \text { where } d_{i}=s_{i j} r_{j}^{\prime 2} Z_{c_{i}}, \text { with } Z_{c_{i}}=\frac{s_{i i}}{r_{i}^{\prime}}\left(\frac{t_{i i}}{t_{j i}}-1\right), \\
a_{0} & =s_{j i} r_{j}^{\prime 2}\left(Z_{c_{i}}-Z\right) Z .
\end{aligned}
$$

By analogy, one can obtain the polynomial coefficients:

$$
\begin{aligned}
& a_{4}^{\prime}=-s_{i j} \alpha_{c}, \\
& a_{3}^{\prime}=-\left(2 s_{i j}-s_{i i}\right) \alpha_{c}, \\
& a_{2}^{\prime}=e_{i}^{\prime} Z-\left(-2 s_{i i}-r_{i}^{\prime} Z+s_{\prime}^{\prime}\right) \alpha_{c} \text { where } e_{i}^{\prime}=s_{i i}^{2} r_{j}^{\prime}+s_{i j} s_{j i} r_{i}^{\prime}>0,
\end{aligned}
$$




$$
\begin{aligned}
& a_{1}^{\prime}=e_{i}^{\prime} Z+\left(s_{i i}+r_{i}^{\prime} Z\right) \alpha_{c}+d_{i}^{\prime} Z \\
& \quad \text { where } d_{i}^{\prime}=s_{j i} r_{i}^{\prime 2} Z_{c_{j}}, \text { with } Z_{c_{j}}=\frac{s_{j j}}{r_{j}^{\prime}}\left(\frac{t_{j j}}{t_{i j}}-1\right) \text {, and } \\
& a_{0}^{\prime}=s_{i j} r_{i}^{\prime 2}\left(Z_{c_{j}}-Z\right) Z .
\end{aligned}
$$

Statement (iv) of Proposition 3.2. Let $\alpha_{c}>0$. Suppose $\alpha_{c}=\alpha_{Z}\left(E^{*}\right)$, this means $\operatorname{det}\left(J\left(E^{*}\right)\right)=0$. Without loss generality, set the eigenvalues are $\lambda_{i 1}=-\left(s_{i i} x_{i}^{*}+s_{j j} x_{j}^{*}\right)$ and $\lambda_{i 2}=0$ associated respectively at eigenvectors: $v_{1}=\left(\theta_{1}, 1\right)^{\prime}$ and respectively $v_{2}=\left(1, \theta_{2}\right)^{\prime}$, where

$$
\theta_{1}=\frac{x_{i}^{*}\left(s_{i i} x_{i}^{*}+s_{i j} x_{j}^{*}+s_{i j}\left(1+x_{j}^{*}\right)\right)}{s_{j j} x_{j}^{*}\left(1+x_{j}^{*}\right)}
$$

and

$$
\theta_{2}=-\frac{s_{j j} x_{j}^{*}+s_{j i} x_{i}^{*}+s_{j i}\left(1+x_{i}^{*}\right)}{s_{j j}\left(1+x_{i}^{*}\right)} .
$$

Let:

$$
P=\left(\begin{array}{cc}
\theta_{1} & 1 \\
1 & \theta_{2}
\end{array}\right) \text { thus } P^{-1}=\frac{1}{1-\theta_{1} \theta_{2}}\left(\begin{array}{cc}
-\theta_{2} & 1 \\
1 & -\theta_{1}
\end{array}\right)
$$

by change

$$
\left(\begin{array}{l}
x_{i} \\
x_{j}
\end{array}\right)=P\left(\begin{array}{l}
x \\
y
\end{array}\right)
$$

we have

$$
\left(\begin{array}{c}
\dot{x} \\
\dot{y}
\end{array}\right)=P^{-1}\left(\begin{array}{c}
\dot{x_{i}} \\
\dot{x_{j}}
\end{array}\right)
$$

which is equivalent to

$$
\begin{gathered}
\left\{\begin{array}{l}
\dot{x}=\frac{1}{1-\theta_{1} \theta_{2}}\left(\dot{x_{j}}-\theta_{2} \dot{x_{i}}\right) \\
\dot{y}=\frac{1}{1-\theta_{1} \theta_{2}}\left(\dot{x}_{i}-\theta_{1} \dot{x}_{j}\right) .
\end{array}\right. \\
\left\{\begin{array}{l}
\dot{x}=\frac{\epsilon_{11} x^{2}+\epsilon_{12} y^{2}+F(x, y)}{1-\theta_{1} \theta_{2}}+o(\|(x, y)\|) \\
\dot{y}=\frac{\epsilon_{21} x^{2}-\epsilon_{22} y^{2}+G(x, y)}{1-\theta_{1} \theta_{2}}+o(\|(x, y)\|)
\end{array}\right.
\end{gathered}
$$


where

$$
\begin{aligned}
\epsilon_{11} & =-s_{j j}+\theta_{1}\left(-s_{j i}+s_{i i} \theta_{1} \theta_{2}+s_{i j} \theta_{2}\right), \\
\epsilon_{21} & =\theta_{1}\left(-s_{i i} \theta_{1}+s_{j i}-s_{j j}+s_{j i} \theta_{1}\right), \\
\epsilon_{12} & =\theta_{2}\left(-s_{j j} \theta_{2}-s_{j i}+s_{i i}+s_{i j} \theta_{2}\right), \\
\epsilon_{22} & =\left(s_{i i}+\theta_{2}\left(s_{i j}-s_{j j} \theta_{1} \theta_{2}-s_{j i} \theta_{1}\right)\right), \\
F(x, y)= & {\left[\left(2 s_{i i} \theta_{1}+s_{i j} \theta_{1} \theta_{2}+s_{i j}\right) \theta_{2}-s_{j i} \theta_{1} \theta_{2}-s_{j i}-2 s_{j j} \theta_{2}\right] x y \quad \text { and } } \\
G(x, y)= & {\left[\left(s_{j i} \theta_{1} \theta_{2}+s_{j i}+2 s_{j j} \theta_{2}\right) \theta_{1}-s_{i j} \theta_{1} \theta_{2}-s_{i j}-2 s_{i i} \theta_{1}\right] x y . }
\end{aligned}
$$

With $F\left(0_{\mathbb{R}^{2}}\right)=G\left(0_{\mathbb{R}^{2}}\right)=0_{\mathbb{R}^{2}}, J Q(0,0)=\left(\begin{array}{ll}0 & 0 \\ 0 & 0\end{array}\right)$ where $J Q$ denotes the Jacobian Matrix of $Q(x, y)=\left(\begin{array}{l}F(x, y) \\ G(x, y)\end{array}\right)$; furthermore, there exists $\delta>0$, $r \geq 1$ and a function $h \in \mathcal{C}^{r}\left(N_{\delta}(0)\right)$ that defines locally the center manifold and satisfies

$$
h(0)=0 \text { and } D h(0)=0 .
$$

So, $y=h(x)=o\left(x^{2}\right)$ in [39]. As $E^{c}=<v_{2}>$ is tangent to the center manifold at origin, then:

$$
W^{c}(0)=E^{c} \text { with } v_{2}=\left(1, \theta_{2}\right)^{\prime} .
$$

Thus $E^{*}$ is stable for the system (2).

\section{B Jacobian Matrix}

— at exclusion equilibrium $E_{i}$ :

$$
J\left(E_{i}\right)=\left(\begin{array}{cc}
-r_{i} Z & -K_{i i} s_{i j}\left(K_{i j}+1\right) \\
0 & \frac{r_{j} Z}{1+K_{i i}}-s_{j i} K_{i i}
\end{array}\right)
$$

The Jacobian Matrix of $Q$ obtained form (22) is

$$
J Q(X, Y)=\left(\begin{array}{cc}
-2 s_{i i} X+\psi Y & \sigma_{1} Y+\psi X \\
-s_{i j} 4 Y & \sigma_{2} Y-s_{j i} X
\end{array}\right)
$$


where $\sigma_{1}=2 \gamma\left(s_{i j}-s_{i i}-s_{j j}\right), \psi=2 s_{i i} \gamma-s_{i j}-s_{j i}$ and $\sigma_{2}=2\left(s_{j i} \gamma-s_{j j}\right)$.

- at coexistence equilibrium $E^{*}$ :

$$
J\left(E^{*}\right)=\left(\begin{array}{cc}
-s_{i i} x_{i}^{*} & \Sigma_{1}\left(x^{*}, y^{*}\right) \\
\Sigma_{2}\left(x^{*}, y^{*}\right) & -s_{j j} x_{j}^{*}
\end{array}\right)
$$

where

$$
\Sigma_{1}\left(x^{*}, y^{*}\right)=-x_{i}^{*}\left(\frac{s_{i i} x_{i}^{*}+s_{i j} x_{j}^{*}}{1+x_{j}^{*}}+s_{i j}\right)
$$

and

$$
\Sigma_{2}\left(x^{*}, y^{*}\right)=-x_{j}^{*}\left(\frac{s_{j j} x_{j}^{*}+s_{j i} x_{i}^{*}}{1+x_{i}^{*}}+s_{j i}\right) .
$$

- at $\left(x_{i}^{\prime}, x_{j}\right)$ where $x_{i}^{\prime}=x_{i}-K_{i i}$ :

$$
\begin{gathered}
J\left(x_{i}^{\prime}, x_{j}\right)=\left(\begin{array}{ll}
j_{1}\left(x_{i}^{\prime}, x_{j}\right) & j_{2}\left(x_{i}^{\prime}, x_{j}\right) \\
j_{3}\left(x_{i}^{\prime}, x_{j}\right) & j_{4}\left(x_{i}^{\prime}, x_{j}\right)
\end{array}\right) \\
j_{1}\left(x_{i}^{\prime}, x_{j}\right)=\frac{-r_{i} Z}{1+x_{j}}-2\left(x_{i}^{\prime}+K_{i i}\right)-s_{i j} x_{j}, \\
j_{2}\left(x_{i}^{\prime}, x_{j}\right)=\frac{-r_{i} Z}{\left(1+x_{j}\right)^{2}}-s_{i j}\left(x_{i}^{\prime}+K_{i i}\right), \\
j_{3}\left(x_{i}^{\prime}, x_{j}\right)=\frac{-r_{j} Z x_{j}}{\left(1+x_{i}^{\prime}+K_{i i}\right)^{2}}-s_{j i} x_{j}, \\
j_{4}\left(x_{i}^{\prime}, x_{j}\right)=\frac{r_{j} Z}{1+x_{i}^{\prime}+K_{i i}}-2 s_{j j} x_{j}-s_{j i}\left(x_{i}^{\prime}+K_{i i}\right) .
\end{gathered}
$$

In particular, when $Z=Z_{c_{i}}$ the jacobian Matrix (B.39) at $x_{i}^{\prime}=0$ and $x_{j}=0$ is as form:

$$
J(0,0)=\left(\begin{array}{cc}
-r_{i} Z & -K_{i i}\left(r_{i} Z+s_{i j}\right) \\
0 & 0
\end{array}\right)
$$

\section{Descartes critera}

Theorem C.1 ([40]). Consider $P_{n}(x)=a_{n} x^{n}+a_{n-1} x^{n-1}+\ldots+a_{0}$ with $a_{n} \neq 0$ a polynomial and $\nu$ the number of sign changes of the coefficients $\left\{a_{i}\right\}_{(i \in \mathbb{N})}$. Then the $k$ number of real positive zero of $P_{n}$ is equal or less than $\nu$ by $(\nu-k)$ is the even number. 


\section{Acknowledgement}

The authors really acknowledge and appreciate the efforts of the unknown reviewers. The second author acknowledges funding from Senegalese Minister of High Education, Research and Innovation through FIRST grant referenced KhadyFIRST2014.

\section{Disclosure of statement}

The research work forms part of the first authors $\mathrm{PhD}$ work, the second co-author is his supervisor and the last authors contribute to competition modeling.

\section{Data Availability of Statement}

The numerical data and hypothetical value of parameters used to support the findings of this research are included within the article. They are either properly assumed or estimated.

\section{Conflict of Interest}

No conflict of interest as far as this research is concerned.

\section{References}

[1] G. Hardin: The competitive exclusion principle. Science, 131, pp: 1292-1297, 1960. https://doi.org/10.1126/science.131.3409.1292

[2] G.F. Gause (1932): Experimental studies on the struggle for existence: I. Mixed population of two species of yeast. Journal of Experimental Biology, 9, pp: 389-402. https://doi.org/10.1242/jeb.9.4.389

[3] V. Volterra (1928): Variations and fluctuations of the number of individuals in animal species living together ICES Journal of Marine Science, 3 (1), pp:351. https://doi.org/10.1093/icesjms/3.1.3

[4] M. Benaïm and C. Lobry (2016): Lotka-volterra with randomly fluctuating environments or "how switching between beneficial environments can make survival harder" The Annals of Applied Probability, 26 (6), pp: 3754-3785. https://doi.org/10.1214/16-AAP1192 
[5] T. Park (1954): Experimental studies of interspecies competition II. Temperature, humidity, and competition in two species of Tribolium. Physiological Zoology, 27 (3), University of Chicago Press, pp: 177-238. https: //www. jstor.org/stable/30152164

[6] G. E. Hutchinson (1961): The paradox of the plankton. American Naturalist, 95 (882), pp: 137-145. https://www.jstor.org/stable/2458386

[7] G. Bunin (2017): Ecological communities with Lotka-Volterra dynamics. American Phytopathological Society, 95 (4), pp: 042-414. https://doi.org/ 10.1103/PhysRevE.95.042414

[8] C. K. Fisher and P. Mehta (2014): The transition between the niche and neutral regimes in ecology. Proceedings of the National Academic Sciences, 111(36), pp: 13111-13116. https://doi.org/10.1073/pnas.1405637111

[9] D. A. Kessler and N. M. Shnerb ( 2015): Generalized model of island biodiversity. Physical Review E, 91 (4), pp: 042-705. https://doi.org/10.1103/ PhysRevE.91.042705

[10] R. M. Sibly, J. Nabe-Nielsen, M. C. Forchhammer, V. E. Forbes and C. J. Topping: The effects of spatial and temporal heterogeneity on the population dynamics of four animal species in a Danish landscape. BMC ecology, 9 (1), 18, 2009. https://doi.org/10.1186/1472-6785-9-18

[11] R. MacArthur (1984): Geographical ecology: patterns in the distribution of species. Princeton University Press.

[12] R. MacArthur and R. Levins (1967): The limiting similarity, convergence, and divergence of coexisting species. The American Naturalist, 101(921), pp: 377-385. https://www. jstor.org/stable/2459090

[13] R. MacArthur, J. M. Diamond, and J. R. Karr (1972): Density compensation in island faunas. Ecology, 53 (2), pp: 330-342. https://doi.org/10.2307/ 1934090

[14] R. MacArthur (1969): Species packing, and what competition minimizes. Proceedings of the National Academy of Sciences, 64 (4), pp: 1369-1371. https://doi.org/10.1073/pnas.64.4.1369

[15] R. MacArthur (1958): Population ecology of some warblers of northeastern coniferous forests. Ecology. 39(4) pp: 599-619. https://doi.org/10.2307/ 1931600

[16] R. MacArthur (1970): Species packing and competitive equilibrium for many species. Theoretical Population Biology, 1(1), pp: 1-11. https://doi.org/ 10.1016/0040-5809 (70) 90039-0 
[17] R. May and R. MacArthur and H. Robert (1972): Niche overlap as a function of environmental variability. Proceedings of the National Academics Sciences, 69, pp: 1109-1113. https://doi.org/10.1073/pnas.69.5.1109

[18] P. Chesson (1990): MacArthur's consumer-resource model. Theoretical Population Biology, 37(1), pp: 26-38. https://doi.org/10.1016/ 0040-5809 (90) 90025-Q

[19] J. Roughgarden (1979): Theory of population genetics and evolutionary ecology: an introduction. Evolution, pp: 1032-1033. https://doi.org/10.1111/ j.1558-5646.1980.tb04043.x

[20] J. Roughgarden (2009): Is there a general theory of community ecology? Biology and Phylosophy, 24, pp: 521-529. https://doi.org/10.1007/ s10539-009-9164-z

[21] J.B. Hutchinson (1947): Notes on the classification and distribution of genera related to Gossypium. New Phytologist, 46(1), pp: 123-141.

[22] D. L. DeAngelis, R. Goldstein and R. V. O'Neill (1975): A model for tropic interaction. Ecology, 56(4), pp: 881-892. https://doi.org/10.2307/1936298

[23] H. L. Smith (2008): The Rosenzweig-MacArthur predator-prey model. School of Mathematical and Statistical Sciences, Arizona State University: Phoenix.

[24] R. Fekih-Salem, T. Sari, N. Abdellatif (2011): Sur un modéle de compétition et de co-existence dans le chémostat, ARIMA, 14, pp: 15-30. https://hal. inria.fr/hal-00777548

[25] P. De Leenheer, D. Angeli and E. D. Sontag (2006): Crowding effects promote coexistence in the chemostat. Journal of Mathematical Analysis and Applications, 319(1), pp: 48-60. https://doi.org/10.1016/j.jmaa.2006.02.036

[26] C. Lobry, J. Harmand (2006): A new hypothesis to explain the coexistence of $n$ species in the presence of a single resource. Comptes Rendus Biologies, 329(1), pp: 40-46. https://doi.org/10.1016/j.crvi.2005.10.004

[27] G.S.K. Wolkowicz, Z. Lu (1998): Direct interference on competition in a chemostat. Journal of Biomathematics, 13(3), pp: 282-291. https:// europepmc.org/article/cba/572445

[28] C. Lobry, A. Rapaport and F. Mazenc (2006): Sur un modéle densitédépendant de compétition sur une ressource Comptes Rendus Biologies. 329, pp: 63-70. https://doi.org/10.1016/j.crvi.2005.11.004

[29] C. Lobry, F. Mazenc, and A. Rapaport (2005): Persistence of ecological models of competition for a single ressource. Comptes rendus mathématique, Academie des Sciences Paris, 1340, pp: 199-204. https://doi.org/10. 1016/j.crma.2004.12.021 
[30] C. Lobry and F. Mazenc (2007): Effet on persistence of intra-specific competition in competition models. Electronic Journal of Differential Equations, 125, pp:1-10. https://eudml.org/doc/128680

[31] N. Kyurkchiev, G. Boyadjiev (2021): Dynamics of modified Lotka-Volterra model with polynomial intervention factors. Methodological aspects. III, International Journal of Differential Equations and Applications, 20(1), pp: 121-132. https://www.ijdea.eu

[32] J. Mallet (2012): The struggle for existence. How the notion of carrying capacity $K$ obscures the links between demography, Darwinian evolution and speciation. Evolutionary Ecology Research, 14, pp: 627-665.

[33] E. Renshaw (1991): Modelling biological populations in space and time. Cambridge Studies in Mathematical Biology, Cambridge University Press, 11. https://doi.org/10.1017/CB09780511624094

[34] E. Barbashin, and N. N. Krasovskii (1961): On stability of motion in the large. Trw Space Technology Labs Los Angeles Calif.

[35] J. Guckenheimer and P. Holmes (1983): Nonlinear Oscillations, Dynamical Systems and Bifurcation of Vector fields. Applied Mathematical Sciences, 42, Springer, New York, NY, pp: 44-45. https://doi.org/10.1007/ 978-1-4612-1140-2

[36] H. R. Thieme (1992): Convergence results and a Poincaré-Bendixson trichotomy for asymptotically autonomous differential equations. Journal of Mathematical Biology, 30(7), pp: 755-763. https://doi.org/10.1007/ BF00173267

[37] N. Kyurkchiev, V. Kyurkchiev, A. Iliev, A. Rahnev (2021): Another Look at SIR/SIRD/SEIR/GSEIR Models: New Trends, Methodological Aspects, Plovdiv University Press, Plovdiv, Bulgaria.

[38] H. L. Smith and P. Waltman (1995): The Theory of the Chemostat - Dynamics of microbial competition. Cambridge University Press, pp: 1-27. https://doi.org/10.1017/CB09780511530043.002

[39] L. Perko (2006): Differential Equations and Dynamical Systems. Texts in Applied Mathematics, 7, Springer, New York, NY. https://doi.org/10. 1007/978-1-4613-0003-8_2

[40] A. G. Khonvaanski $\tilde{i}$ (1991): Fewnomials, 88, Translations of Mathematical Monographs. 